\title{
Pauli Approximations to the Self-Adjoint Extensions of the Aharonov-Bohm Hamiltonian
}

\author{
J.L. Borg ${ }^{a b c}$ and J.V. Pulé ${ }^{a d ~ e}$
}

\begin{abstract}
It is well known that the formal Aharonov-Bohm Hamiltonian operator, describing the interaction of a charged particle with a magnetic vortex, has a four-parameter family of self-adjoint extensions, which reduces to a two-parameter family if one requires that the Hamiltonian commutes with the angular momentum operator. The question we study here is which of these self-adjoint extensions can considered as limits of regularised Aharonov-Bohm Hamiltonians, that is Pauli Hamiltonians in which the magnetic field corresponds to a flux tube of non-zero diameter. We show that not all the self-adjoint extensions in this two-parameter family can be obtained by these approximations, but only two one-parameter subfamilies. In these two cases we can choose the gyromagnetic ratio in the approximating Pauli Hamiltonian in such a way that we get convergence in the norm resolvent sense to the corresponding self-adjoint extension.
\end{abstract}

${ }^{a}$ Department of Mathematical Physics, University College Dublin (National University of Ireland, Dublin), Belfield, Dublin 4, Ireland.

${ }^{\mathrm{b}}$ e-mail: james.borg@um.edu.mt

${ }^{c}$ on leave of absence from Department of Mathematics, University of Malta, Msida MSD 06, Malta.

de-mail: Joe.Pule@ucd.ie

${ }^{\mathrm{e}}$ Research Associate, School of Theoretical Physics, Dublin Institute for Advanced Studies. 


\section{Introduction}

The Aharonov-Bohm Hamiltonian operator, describing the interaction of a charged particle with a magnetic vortex, that is, an infinitely extended, infinitely thin, impenetrable magnetic flux tube, is given by

$$
H=\frac{1}{2 \mathrm{~m}}\left(\mathbf{p}-\frac{e}{c} \mathbf{A}\right)^{2}
$$

where the vector potential $\mathbf{A}$ is given by

$$
\mathbf{A}=\frac{\phi}{2 \pi} \frac{\mathbf{k} \times \mathbf{r}}{r^{2}}
$$

$\phi$ being the flux of the tube. It is well known $[1,2]$ that this formal operator has a fourparameter family of self-adjoint extensions, which reduces to a two-parameter family if one requires that the Hamiltonian commutes with the angular momentum operator. These selfadjoint extensions can be obtained formally by adding a delta-function. The question we study in this paper is which of these self-adjoint extensions can considered as limits of regularised Aharonov-Bohm Hamiltonians, that is Pauli Hamiltonians in which the magnetic field corresponds to a flux tube of non-zero diameter.

This problem has been studied by Bordag and Voropaev [3] and by Moroz [4]. These authors make the connection between the regularised Hamiltonian and the self-adjoint extensions of the A-B Hamiltonian and show that the gyromagnetic ratio has to be chosen in a particular way. They do this by by matching the bound states as the radius of the vortex tends to zero, but do not prove convergence of the operators. Also they do not take into account the second parameter in the two-parameter family of self-adjoint extensions mentioned above. Here we proceed more sytematically to extend the results of [3] and [4]. We consider convergence in the norm resolvent sense. We show that not all the self-adjoint extensions in this twoparameter family can be obtained by these approximations, but only two one-parameter subfamilies. Tamura [5] has done related work but with a different emphasis.

When the A-B Hamiltonian is decomposed into the subspaces corresponding to the values of the angular momentum $m \in \mathbb{Z}$, it turns out that if $N$ is the integer part of the dimensionless parameter $\alpha=\phi e / h c$, then the Hamiltonians restricted to $m=N$ and $m=N+1$ are not essentially self-adjoint while the ones with other values of $m$ are essentially self-adjoint. The operators corresponding to $m=N$ and $m=N+1$ each have a one-parameter family of selfadjoint extensions. We denote these parameters in $(-\infty, \infty]$ by $\nu_{N}$ and $\nu_{N+1}$ respectively, $\nu_{N}=\infty$ and $\nu_{N+1}=\infty$ corresponding to the regular self-adjoint extension. We prove that for the subfamilies $\nu_{N} \in(-\infty, \infty), \nu_{N+1}=\infty$ and $\nu_{N+1} \in(-\infty, \infty), \nu_{N}=\infty$ we can choose the gyromagnetic ratio, $g$, in the approximating Pauli Hamiltonian in such a way that we get convergence in the norm resolvent sense to the corresponding self-adjoint extension. The approximating Hamiltonian is

$$
H_{R}=\frac{1}{2 \mathrm{~m}}\left(\mathbf{p}-\frac{e}{c} \mathbf{A}_{R}\right)^{2}-\frac{g e \hbar}{2 \mathrm{~m} c} \mathbf{k} \cdot \mathbf{B}_{R}
$$

with $\mathbf{B}_{R}=$ curl $\mathbf{A}_{R}$. The vector potential $\mathbf{A}_{R}$ is 0 inside a tube of radius $R$ away from its boundary and given by (1.2) outside the tube away from the boundary. It was shown in [3] and [4] that to obtain a non-trivial limit, $g$ must depend on $R$ and must tend to 2 in a certain way. For a discussion of the physical significance of this limit we refer the reader to these 
papers. The same result holds here for the self-adjoint extensions with $\nu_{N+1} \in(-\infty, \infty)$, $\nu_{N}=\infty$. However, for the self-adjoint extensions with $\nu_{N+1} \in(-\infty, \infty), \nu_{N}=\infty$, which were not considered in [3] and [4], $g$ must behave like $-2+4(N+1) / \alpha$.

Two other approximations have been considered, namely, the case when the magnetic field inside the tube is homogeneous [3,4] and the case when it is proportional to $1 / r$ [3]. The situation in these cases is similar but more complex. We deal with these briefly at the end of the paper.

The paper is set out as follows. In Section 2 we give the basic properties of the A-B Hamiltonian. In Section 3 we carry out the approximation to the A-B Hamiltonian with an infinitely thin infinitely extended cylindrical shell of non-zero radius $R$. In Section 4 we smooth the flux shell to give it a non-zero thickness. In this section we only sketch the proof. In Section 5 we discuss the other two approximations. In the Appendix we give the asymptotic behaviour of the Special Functions needed for these approximations.

\section{The A-B Hamiltonian}

In the sequel we set $\hbar^{2} / \mathrm{m}=2$ and $e / c=1$ so that the A-B Hamiltonian is formally the operator

$$
H=(i \nabla+\mathbf{A})^{2},
$$

in $L^{2}\left(\mathbb{R}^{2}\right)$, where the vector potential $\mathbf{A}$ is now given by

$$
\mathbf{A}=\alpha \frac{\mathbf{k} \times \mathbf{r}}{r^{2}}
$$

We let $\alpha=N+\delta$, where $N \in \mathbb{Z}$ and $0<\delta<1$. Without loss of generality, we shall assume that $\alpha>0$.

This Hamiltonian is discussed in great detail in [1] and [2]. The analysis proceeds by decomposing the underlying space and studying the radial Hamiltonians

$$
h_{m}=-\frac{1}{r} \frac{\partial}{\partial r} r \frac{\partial}{\partial r}+\frac{(m-\alpha)^{2}}{r^{2}}
$$

in $L^{2}((0, \infty), r d r)$. Taking as domain $C_{0}^{\infty}((0, \infty), r d r)$, these operators are essentially selfadjoint, except for the cases $m=N, N+1$ which have deficiency indices $(1,1)$. These two operators therefore have self-adjoint extensions $h_{N, \nu_{N}}$ and $h_{N+1, \nu_{N+1}}$, parametrized by $\nu_{N}$ and $\nu_{N+1}$, where $-\infty<\nu_{N}, \nu_{N+1} \leqslant \infty$. These self-adjoint extensions can be identified with the boundary conditions

$$
\nu_{m} \phi_{0}=\phi_{1}
$$

where

$$
\phi_{0}=\lim _{r \downarrow 0} r^{|m-\alpha|} \phi(r)
$$

and

$$
\phi_{1}=\lim _{r \downarrow 0} r^{-|m-\alpha|}\left[\phi(r)-r^{-|m-\alpha|} \phi_{0}\right] .
$$

A four-parameter family of self-adjoint extensions of $H^{A B}$ can be constructed from these. If we consider only self-adjoint extensions which commute with the angular momentum 
operator, this reduces to a two-parameter family. For this particular choice, the self-adjoint extension, $H^{\nu}$, with $\boldsymbol{\nu} \in(-\infty, \infty]^{2}$, is just the direct sum

$$
H^{\nu}=h_{N, \nu_{N}} \oplus h_{N+1, \nu_{N+1}} \oplus \bigoplus_{\substack{m=-\infty \\ m \neq N, N+1}}^{\infty} h_{m}
$$

We shall write $H^{\infty}$ for $H^{\boldsymbol{\nu}}$ with $\boldsymbol{\nu}=(\infty, \infty)$. Let $g_{k, m}=\left(h_{m}-k^{2}\right)^{-1}$. Then

$$
g_{k, m}\left(r, r^{\prime}\right)=\frac{i \pi}{2} J_{|m-\alpha|}\left(k r_{<}\right) H_{|m-\alpha|}^{(1)}\left(k r_{>}\right),
$$

where $r_{<}=\min \left\{r, r^{\prime}\right\}$ and $r_{>}=\max \left\{r, r^{\prime}\right\}$. The resolvents $g_{k, m}^{\nu_{m}}=\left(h_{m, \nu_{m}}-k^{2}\right)^{-1}, m=$ $N, N+1$, are given by

$$
g_{k, m}^{\nu m}\left(r, r^{\prime}\right)=g_{k, m}\left(r, r^{\prime}\right)+c\left(m, \nu_{m}, k\right) H_{|m-\alpha|}^{(1)}(k r) H_{|m-\alpha|}^{(1)}\left(k r^{\prime}\right), m=N, N+1,
$$

with

$$
\begin{aligned}
c(m, \nu, k)= & \frac{-\pi^{2}}{2 \Gamma(1+|m-\alpha|)}\left(\frac{k}{2}\right)^{2|m-\alpha|} \\
& \times\left(e^{-i \pi|m-\alpha|} \frac{\Gamma(1-|m-\alpha|)}{|m-\alpha|}\left(\frac{k}{2}\right)^{2|m-\alpha|}+\nu \Gamma(|m-\alpha|)\right)^{-1} \\
c(m, \infty, k)= & 0 .
\end{aligned}
$$

Finally, the resolvent of the Hamiltonian $G_{k}^{\boldsymbol{\nu}}=\left(H^{\boldsymbol{\nu}}-k^{2}\right)^{-1}$ is given by (see [1]):

$$
G_{k}^{\nu}=g_{k, N}^{\nu_{N}} \oplus g_{k, N+1}^{\nu_{N+1}} \oplus \bigoplus_{\substack{m=-\infty \\ m \neq N, N+1}}^{\infty} g_{k, m}
$$

Note that the operators $h_{m, \nu_{m}}$ have one bound state, $E_{m}$, given by

$$
\left(\frac{\sqrt{E_{m}}}{2}\right)^{2|m-\alpha|}=-\frac{\Gamma(1+|m-\alpha|)}{\Gamma(1-|m-\alpha|)} \nu_{m}, \quad m=N, N+1 .
$$

\section{Approximation by Finite Flux Tube}

The Aharonov-Bohm Hamiltonian consists of an infinitely thin magnetic flux tube. As a first approximation to $H^{A B}$, consider a flux tube of radius $R>0$, with a $\delta$-function on a cylindrical shell (following [3] but see also [6], [4]). That is we take the vector potential

$$
\mathbf{A}_{R}= \begin{cases}0 & r<R \\ \alpha \frac{\mathbf{k} \times \mathbf{r}}{r^{2}} & r>R\end{cases}
$$

Then $\mathbf{k} \cdot \mathbf{B}_{R}=\frac{\alpha}{R} \delta(r-R)$, so that formally, the Hamiltonian is then given by:

$$
H_{R}=\left(i \nabla+\mathbf{A}_{R}\right)^{2}+\frac{\beta}{R} \delta(r-R)
$$


where $\beta=-g \alpha / 2$. The components in $L^{2}((0, \infty), r d r)$ corresponding to the angular momentum $m$ of this formal operator are

$$
-\frac{1}{r} \frac{\partial}{\partial r} r \frac{\partial}{\partial r}+\frac{(m-\alpha \Theta(r-R))^{2}}{r^{2}}+\frac{\beta}{R} \delta(r-R)
$$

where $\Theta$ is the unit step function. The procedure for adding a point interaction at $r=R$ to a radial Hamiltonian is standard (cfr. [7] Sect. I.3.1). The point we make here is that $\beta$, the strength of the point interaction, has to depend on $R$ and $\alpha$ in a definite way so that the self-adjoint extensions obtained in this manner converge to the A-B self-adjoint extensions as $R$ tends to 0 .

Consider the following operator in $L^{2}((0, \infty), r d r)$

$$
h_{m, R}=-\frac{1}{r} \frac{\partial}{\partial r} r \frac{\partial}{\partial r}+\frac{(m-\alpha \Theta(r-R))^{2}}{r^{2}}
$$

with the closure of $C_{0}^{\infty}((0, \infty) \backslash\{R\})$ as its domain ([7], p. 75) i.e.

$$
\begin{aligned}
\mathcal{D}\left(h_{m, R}\right)= & \left\{g \in H^{2,2}((0, \infty), r d r) \text { s.t. } h_{m, R} g \in L^{2}((0, \infty), r d r)\right. \\
& \text { and } g(R)=0\} .
\end{aligned}
$$

Then its adjoint has domain ([7], p. 75)

$$
\begin{aligned}
\mathcal{D}\left(h_{m, R}^{*}\right)= & \left\{g \in H^{2,2}((0, \infty) \backslash\{R\}, r d r) \cap H^{2,1}((0, \infty), r d r)\right. \\
& \text { s.t. } \left.h_{m, R} g \in L^{2}((0, \infty), r d r)\right\} .
\end{aligned}
$$

The equation $h_{m, R}^{*} \phi=k^{2} \phi, \Im(k)>0$, has one solution in $\mathcal{D}\left(h_{m, R}^{*}\right)$ for all values of $m \in \mathbb{Z}$ (see Note 1, Appendix 1), given by

$$
\phi_{m, k}(r)= \begin{cases}J_{|m|}(k r) & r<R \\ B_{m}(k) H_{|m-\alpha|}^{(1)}(k r) & r>R\end{cases}
$$

where $B_{m}(k)$ is chosen so that the condition $\phi_{m, k}(R+)=\phi_{m, k}(R-)$ is satisfied. Thus the operators $h_{m, R}$ have deficiency indices $(1,1)$. Self-adjoint extensions $h_{m, R}^{\beta}$ are obtained by imposing the following boundary condition on the domain of $h_{m, R}$ :

$$
\phi_{m, k}^{\prime}(R+)-\phi_{m, k}^{\prime}(R-)=\frac{\beta(\alpha, R)}{R} \phi_{m, k}(R),
$$

where $\beta(\alpha, R)$ is a constant parameter ([7] p. 76). Note that we do not want the parameter $\beta(\alpha, R)$ to depend on $m$ since it represents $-g \alpha / 2$. We shall henceforth be writing $\beta$ for this parameter to make the notation less cumbersome.

Next we find the resolvent $g_{k, m, R}^{\beta}=\left(h_{m, R}^{\beta}-k^{2}\right)^{-1}$. Note first that $g_{k, m, R}^{0}=\left(h_{m, R}-k^{2}\right)^{-1}$, $\Im(k)>0$, is given by (Note 2 , Appendix 1 )

$$
g_{k, m, R}^{0}\left(r, r^{\prime}\right)= \begin{cases}\frac{i \pi}{2} J_{|m|}\left(k r_{<}\right)\left(\frac{A_{m, R}^{(2)}(k)}{B_{m, R}^{(2)}(k)} J_{|m|}\left(k r_{>}\right)+H_{|m|}^{(1)}\left(k r_{>}\right)\right), & r, r^{\prime}<R \\ \frac{i \pi}{2}\left(J_{|m-\alpha|}\left(k r_{<}\right)+\frac{B_{m, R}^{(1)}(k)}{A_{m, R}^{(1)}(k)} H_{|m-\alpha|}^{(1)}\left(k r_{<}\right)\right) H_{|m-\alpha|}^{(1)}\left(k r_{>}\right), r, r^{\prime}>R \\ \frac{i \pi}{2 A_{m, R}^{(1)}(k)} J_{|m|}\left(k r_{<}\right) H_{|m-\alpha|}^{(1)}\left(k r_{>}\right) & \text {otherwise }\end{cases}
$$


where the constants $A_{m, R}^{(1)}(k), A_{m, R}^{(2)}(k), B_{m, R}^{(1)}(k)$ and $B_{m, R}^{(2)}(k)$ are given by:

$$
\begin{aligned}
A_{m, R}^{(1)}(k) & =\frac{W\left[J_{|m|}, H_{|m-\alpha|}^{(1)}\right](k R)}{W\left[J_{|m-\alpha|}, H_{|m-\alpha|}^{(1)}\right](k R)}, \\
B_{m, R}^{(1)}(k) & =\frac{W\left[J_{|m|}, J_{|m-\alpha|}\right](k R)}{W\left[H_{|m-\alpha|}^{(1)}, J_{|m-\alpha|}\right](k R)}, \\
A_{m, R}^{(2)}(k) & =\frac{W\left[H_{|m-\alpha|}^{(1)}, H_{|m|}^{(1)}\right](k R)}{W\left[J_{|m|}, H_{|m|}^{(1)}\right](k R)}, \\
B_{m, R}^{(2)}(k) & =\frac{W\left[J_{|m|}, H_{|m-\alpha|}^{(1)}\right](k R)}{W\left[J_{|m|}, H_{|m|}^{(1)}\right](k R)} .
\end{aligned}
$$

Here $W[\cdot, \cdot]$ denotes the Wronskian. Then the resolvent of $h_{m, R}^{\beta}$ is given by (Note 3, Appendix 1):

$$
g_{k, m, R}^{\beta}=g_{k, m, R}^{0}-\frac{\beta}{1+\beta g_{k, m, R}^{0}(R, R)} g_{k, m, R}^{0}(\cdot, R) \otimes \overline{g_{k, m, R}^{0}(\cdot, R)},
$$

for $\Im(k)>0$. We are interested in the behaviour of $g_{k, m, R}^{\beta}\left(r, r^{\prime}\right)$ for small $R$. We note first that $\lim _{R \rightarrow 0} g_{k, m, R}^{0}\left(r, r^{\prime}\right)=g_{k, m}\left(r, r^{\prime}\right)$, which is the resolvent of the regular operator. For small $R$,

$$
g_{k, m, R}^{0}(r, R) g_{k, m, R}^{0}\left(R, r^{\prime}\right) \simeq \tilde{c}_{m}^{2}(k) R^{2|m-\alpha|} H_{|m-\alpha|}^{(1)}(k r) H_{|m-\alpha|}^{(1)}\left(k r^{\prime}\right),
$$

where

$$
\tilde{c}_{m}(k)=\frac{i \pi}{(|m-\alpha|+|m|) \Gamma(|m-\alpha|)}\left(\frac{k}{2}\right)^{|m-\alpha|} .
$$

If $|m-\alpha|>1$ (i.e. if $m \notin\{N, N+1\}$ ), then the second term will either go to zero, or to a constant multiple of $H_{|m-\alpha|}^{(1)}(k r) H_{|m-\alpha|}^{(1)}\left(k r^{\prime}\right)$. Now $H_{|m-\alpha|}^{(1)}(k r)$ is not in $L^{2}((0, \infty), r d r)$ since it behaves like $r^{-|m-\alpha|}$ for small $r$ (see Note 1, Appendix 1). So in the latter case, the limit is not the kernel of a rank-one operator in $L^{2}((0, \infty), r d r)$. In particular, this means that it cannot be the kernel of a resolvent operator. Thus a meaningful non-zero limit for the second term in equation (3.14) exists only for the cases $m=N, N+1$. It shall be shown later that for small $R$, we get

$$
g_{k, m, R}^{0}(R, R) \simeq \frac{1}{|m-\alpha|+|m|}\left(1-\frac{2 e^{-i \pi|m-\alpha|} \Gamma(1-|m-\alpha|)}{(|m-\alpha|+|m|) \Gamma(|m-\alpha|)}\left(\frac{k R}{2}\right)^{2|m-\alpha|}\right) .
$$

For the case $r, r^{\prime}>R$ (the other cases are straightforward), this results in

$$
g_{k, m, R}^{\beta}\left(r, r^{\prime}\right) \simeq g_{k, m, R}^{0}\left(r, r^{\prime}\right)-\frac{\beta \tilde{c}_{m}^{2}(k) R^{2|m-\alpha|}}{1+\frac{\beta\left(1-\tilde{c}^{\prime}(k) R^{2|m-\alpha|}\right)}{|m-\alpha|+|m|}} H_{|m-\alpha|}^{(1)}(k r) H_{|m-\alpha|}^{(1)}\left(k r^{\prime}\right),
$$

where

$$
\tilde{c}^{\prime}(k)=\frac{2 e^{-i \pi|m-\alpha|} \Gamma(1-|m-\alpha|)}{(|m-\alpha|+|m|) \Gamma(|m-\alpha|)}\left(\frac{k}{2}\right)^{2|m-\alpha|} .
$$


The second term in (3.18) converges to a non-zero limit if $\beta$ has the following $R$-dependence for small $R$ :

$$
\beta \simeq-(|m-\alpha|+|m|)\left(1-\frac{2|m-\alpha| \nu_{m}}{|m-\alpha|+|m|} R^{2|m-\alpha|}\right)
$$

Then we obtain

$$
\lim _{R \rightarrow 0} g_{k, m, R}^{\beta}\left(r, r^{\prime}\right)=g_{k, m}\left(r, r^{\prime}\right)+c\left(m, \nu_{m}, k\right) H_{|m-\alpha|}^{(1)}(k r) H_{|m-\alpha|}^{(1)}\left(k r^{\prime}\right) .
$$

This gives the correct expression in (2.5) for the kernel of the resolvent of some self-adjoint extension of $h_{m}, m=N, N+1$.

The following are the only cases of interest:

(I) if $\beta \simeq-\alpha\left(1-\frac{2 \delta}{\alpha} \nu_{N} R^{2 \delta}\right)$, then the second term in equation (3.14) approaches

1. a non-zero limit for $m=N$, corresponding to the self-adjoint extension $h_{N, \nu_{N}}$;

2. zero limit for $m=N+1$, corresponding to the regular self-adjoint extension $h_{N+1, \infty}$;

3. zero limit for $m \neq N, N+1$, corresponding to the self-adjoint operators $h_{m}$.

(II) if $\beta \simeq(\alpha-2(N+1))\left(1-\frac{2(1-\delta)}{2(N+1)-\alpha} \nu_{N+1} R^{2(1-\delta)}\right)$, then the second term in equation (3.14) approaches

1. a non-zero limit for $m=N+1$, corresponding to the self-adjoint extension $h_{N+1, \nu_{N+1}}$;

2. zero limit for $m=N$, corresponding to the regular self-adjoint extension $h_{N, \infty}$;

3. zero limit for $m \neq N, N+1$, as before.

We can state the above results as a Theorem:

\section{Theorem 1}

Let

$$
H_{R}^{\beta}=\bigoplus_{m=-\infty}^{\infty} h_{m, R}^{\beta} .
$$

Then $H_{R}^{\beta(\alpha, R)}$ converges, as $R \rightarrow 0$, to one of the self-adjoint extensions $H^{\nu}$ of the $A$-B Hamiltonian only if either

(I) $\frac{\beta(\alpha, R)+\alpha}{R^{2 \delta}} \rightarrow 2 \delta \nu_{N}$

(II) $\frac{\beta(\alpha, R)-\alpha+2(N+1)}{R^{2(1-\delta)}} \rightarrow 2(1-\delta) \nu_{N+1}$.

In case $(\mathrm{I}) H_{R}^{\beta(\alpha, R)}$ converges in the norm resolvent sense, as $R \rightarrow 0$, to $H^{\left(\nu_{N}, \infty\right)}$, and in case (II) to $H^{\left(\infty, \nu_{N+1}\right)}$. 
Proof Let us consider case (I), case (II) is similar. Since

$$
\begin{aligned}
& \left\|\left(H_{R}^{\beta(\alpha, R)}-k^{2}\right)^{-1}-G_{k}^{\left(\nu_{N}, \infty\right)}\right\| \\
& =\max \left\{\left\|g_{k, N, R}^{\beta(\alpha, R)}-g_{k, N}^{\nu_{N}}\right\|,\left\|g_{k, N+1, R}^{\beta(\alpha, R)}-g_{k, N+1}\right\|, \sup _{\substack{m \in \mathbb{Z} \\
m \neq N, N+1}}\left\|g_{k, m, R}^{\beta(\alpha, R)}-g_{k, m}\right\|\right\},
\end{aligned}
$$

we not only have to prove that the the terms in the righthand side of the above equations tend to zero, but we have to show that $\left\|g_{k, m, R}^{\beta(\alpha, R)}-g_{k, m}\right\|$ tends to zero uniformly in $m$. To do this we need to obtain detailed upper and lower bounds on the special functions $J_{\nu}$ and $H_{\nu}^{(1)}$. These are given in the Appendix.

The first term in the expressions for $g_{k, m, R}^{\beta(\alpha, R)}$ is $g_{k, m, R}^{0}$ and first term in the expressions for $g_{k, m}^{\nu}$ is $g_{k, m}$. Therefore we start with the following lemma. Here $\|\cdot\|_{2}$ denotes the Hilbert-Schmidt norm, and of course $\|\cdot\| \leqslant\|\cdot\|_{2}$.

\section{Lemma 1}

For any $m, \lim _{R \rightarrow 0}\left\|g_{k, m, R}^{0}-g_{k, m}\right\|_{2}=0$.

Furthermore, there exists $M \in \mathbb{N}$ such that for $|m|>M$, there exists a constant $c(R)$, independent of $m$, such that $\left\|g_{k, m, R}^{0}-g_{k, m}\right\|_{2} \leqslant c(R)$, and $\lim _{R \rightarrow 0} c(R)=0$.

\section{Proof :}

$$
\left\|g_{k, m, R}^{0}-g_{k, m}\right\|_{2} \leqslant\left\|\widetilde{g}_{1}\right\|_{2}+\left\|\widetilde{g}_{2}\right\|_{2}+\left\|\widetilde{g}_{3}\right\|_{2}+\left\|\widetilde{g}_{4}\right\|_{2}+\left\|\widetilde{g}_{5}-g_{k, m}\right\|_{2}
$$

where

$$
\begin{aligned}
& \widetilde{g}_{1}\left(r, r^{\prime}\right)=\frac{i \pi}{2} \frac{A_{m, R}^{(2)}(k)}{B_{m, R}^{(2)}(k)} J_{|m|}(k r) J_{|m|}\left(k r^{\prime}\right) \mathbf{1}_{(0, R) \times(0, R)}\left(r, r^{\prime}\right) \\
& \widetilde{g}_{2}\left(r, r^{\prime}\right)=\frac{i \pi}{2} J_{|m|}\left(k r_{<}\right) H_{|m|}^{(1)}\left(k r_{>}\right) \mathbf{1}_{(0, R) \times(0, R)}\left(r, r^{\prime}\right) \\
& \widetilde{g}_{3}\left(r, r^{\prime}\right)=\frac{i \pi}{2} \frac{B_{m, R}^{(1)}(k)}{A_{m, R}^{(1)}(k)} H_{|m-\alpha|}^{(1)}(k r) H_{|m-\alpha|}^{(1)}\left(k r^{\prime}\right) \mathbf{1}_{(R, \infty) \times(R, \infty)}\left(r, r^{\prime}\right) \\
& \widetilde{g}_{4}\left(r, r^{\prime}\right)=\frac{i \pi}{2 A_{m, R}^{(1)}(k)} J_{|m|}\left(k r_{<}\right) H_{|m-\alpha|}^{(1)}\left(k r_{<}\right) \mathbf{1}_{(0, R] \times[R, \infty) \cup[R, \infty) \times(0, R]}\left(r, r^{\prime}\right) \\
& \widetilde{g}_{5}\left(r, r^{\prime}\right)=\frac{i \pi}{2} J_{|m-\alpha|}\left(k r_{<}\right) H_{|m-\alpha|}^{(1)}\left(k r_{>}\right) \mathbf{1}_{(R, \infty) \times(R, \infty)}\left(r, r^{\prime}\right)
\end{aligned}
$$

Using the bounds (7.32), (7.41)and (7.45) in Appendix 2 and the relation

$$
W\left[J_{\nu}(z), H_{\nu}^{(1)}(z)\right]=\frac{2 i}{\pi z},
$$

we can see that for any $\varepsilon>0$, there exists $R_{0}>0$ such that if $R<R_{0}$, then the following 
bounds hold:

$$
\begin{aligned}
& \frac{1}{\left|A_{m, R}^{(1)}(k)\right|} \leqslant \frac{2 \Gamma(|m|+1)}{|| m-\alpha|+| m|| \Gamma(|m-\alpha|)}\left|\frac{k R}{2}\right|^{|m-\alpha|-|m|}(1+\varepsilon) \\
& \left|\frac{B_{m, R}^{(1)}(k)}{A_{m, R}^{(1)}(k)}\right| \leqslant \frac{\pi|| m-\alpha|-| m||}{(|m-\alpha|+|m|) \Gamma(|m-\alpha|) \Gamma(|m-\alpha|+1)}\left|\frac{k R}{2}\right|^{2|m-\alpha|}(1+\varepsilon) \\
& \left|\frac{A_{m, R}^{(2)}(k)}{B_{m, R}^{(2)}(k)}\right| \leqslant \frac{|| m-\alpha|-| m|| \Gamma(|m|) \Gamma(|m|+1)}{2 \pi(|m-\alpha|+|m|)}\left|\frac{k R}{2}\right|^{-2|m|}(1+\varepsilon)
\end{aligned}
$$

Now we can find bounds for the terms in equation (3.24) for small $R$.

$$
\left\|\widetilde{g}_{1}\right\|_{2}^{2}=\iint\left|\widetilde{g}_{1}\left(r, r^{\prime}\right)\right|^{2} r d r r^{\prime} d r^{\prime}=\frac{\pi^{2}}{4}\left|\frac{A_{m, R}^{(2)}(k)}{B_{m, R}^{(2)}(k)}\right|^{2}\left(\int_{0}^{R} r d r\left|J_{|m|}(k r)\right|^{2}\right)^{2} .
$$

Using (3.33) and (7.1) we get

$$
\left\|\widetilde{g}_{1}\right\|_{2}^{2} \leqslant \begin{cases}\frac{(|m-\alpha|-|m|)^{2} R^{4}}{64|m|^{2}(|m|+1)^{2}(|m-\alpha|+|m|)^{2}}(1+\varepsilon), & m \neq 0, \\ \frac{R^{4}}{64}(1+\varepsilon), & m=0 .\end{cases}
$$

Next we have

$$
\begin{aligned}
\left\|\widetilde{g}_{2}\right\|_{2}^{2} & =\int\left|\widetilde{g}_{2}\left(r, r^{\prime}\right)\right|^{2} r d r r^{\prime} d r^{\prime} \\
& =\frac{\pi^{2}}{4} \int_{0}^{R} r d r r^{\prime} d r^{\prime}\left|J_{|m|}\left(k r_{<}\right)\right|^{2}\left|H_{|m|}^{(1)}\left(k r_{>}^{\prime}\right)\right|^{2} \\
& =\frac{\pi^{2}}{2} \int_{0}^{R} r d r\left|J_{|m|}(k r)\right|^{2} \int_{r}^{R} r^{\prime} d r^{\prime}\left|H_{|m|}^{(1)}\left(k r^{\prime}\right)\right|^{2} .
\end{aligned}
$$

From (7.1), (7.18) and (7.25), we get

$$
\left\|\widetilde{g}_{2}\right\|_{2}^{2} \leqslant \begin{cases}\frac{R^{4}}{8|m|^{2}(1+|m|)}(1+\varepsilon), & |m|>1, \\ \frac{R^{4}}{32}(1+\varepsilon), & |m|=1, \\ \frac{R^{4}}{32}\left(8 \ln \left|\frac{k}{2}\right|+14 \ln R+1\right)(1+\varepsilon), & m=0 .\end{cases}
$$

From the relations $\overline{K_{\nu}(z)}=K_{\nu}(\bar{z})$ and

$$
H_{\nu}^{(1)}(z)=-\frac{2 i}{\pi} e^{-\frac{1}{2} i \pi \nu} K_{\nu}(-i z),
$$


we obtain

$$
\begin{array}{r}
\int_{R}^{\infty} r d r\left|H_{|m-\alpha|}^{(1)}(k r)\right|^{2}=\frac{4}{\pi^{2}} \int_{R}^{\infty} d r \frac{r^{2|m-\alpha|+\xi-1}}{r^{2|m-\alpha|+\xi-2}} K_{|m-\alpha|}(i \bar{k} r) K_{|m-\alpha|}(-i k r) \\
\leqslant \frac{4 R^{2-2|m-\alpha|-\xi}}{\pi^{2}} \int_{0}^{\infty} d r r^{2|m-\alpha|+\xi-1} K_{|m-\alpha|}(i \bar{k} r) K_{|m-\alpha|}(-i k r) .
\end{array}
$$

for $2|m-\alpha|+\xi>2$.

Using the formula 6.576 of [9], we then get

$$
\begin{gathered}
\int_{R}^{\infty} r d r\left|H_{|m-\alpha|}^{(1)}(k r)\right|^{2} \leqslant \frac{2}{\pi^{2}}|k|^{-2|m-\alpha|-\xi}\left|\frac{R}{2}\right|^{2-2|m-\alpha|-\xi} \frac{\Gamma\left(\frac{\xi}{2}\right) \Gamma\left(\frac{1}{2}\right)}{\Gamma\left(\frac{\xi}{4}+\frac{1}{2}\right)} \times \\
\times \frac{\Gamma\left(|m-\alpha|+\frac{\xi}{2}+\frac{1}{2}\right) \Gamma\left(2|m-\alpha|+\frac{\xi}{2}\right)\left(\Gamma\left(|m-\alpha|+\frac{\xi}{2}\right)\right)^{2}}{\Gamma\left(|m-\alpha|+\frac{\xi}{4}+\frac{1}{2}\right) \Gamma(2|m-\alpha|+\xi)}
\end{gathered}
$$

for $|m-\alpha| \geqslant 1$ and $\xi>0$.

So, if $m \neq N, N+1$,

$$
\begin{aligned}
\left\|\widetilde{g}_{3}\right\|_{2}^{2}= & \int\left|\widetilde{g}_{3}\left(r, r^{\prime}\right)\right|^{2} r d r r^{\prime} d r^{\prime} \\
= & \frac{\pi^{2}}{4}\left|\frac{B_{m, R}^{(1)}(k)}{A_{m, R}^{(1)}(k)}\right|^{2} \int_{R}^{\infty} r d r\left|H_{|m-\alpha|}^{(1)}(k r)\right|^{2} \int_{r}^{\infty} r^{\prime} d r^{\prime}\left|H_{|m-\alpha|}^{(1)}\left(k r^{\prime}\right)\right|^{2} \\
\leqslant & \frac{\pi^{2}}{4}\left|\frac{B_{m, R}^{(1)}(k)}{A_{m, R}^{(1)}(k)}\right|^{2}\left[\int_{R}^{\infty} r d r\left|H_{|m-\alpha|}^{(1)}(k r)\right|^{2}\right]^{2} \\
\leqslant & \frac{(|m-\alpha|-|m|)^{2}}{(|m-\alpha|+|m|)^{2}}\left(\frac{R}{2}\right)^{4-2 \xi}|k|^{-2 \xi}\left(\frac{\Gamma\left(\frac{\xi}{2}\right) \Gamma\left(\frac{1}{2}\right)}{\Gamma\left(\frac{\xi}{4}+\frac{1}{2}\right)}\right)^{2}(1+\varepsilon) \times \\
& \times\left(\frac{\Gamma\left(|m-\alpha|+\frac{\xi}{2}+\frac{1}{2}\right) \Gamma\left(2|m-\alpha|+\frac{\xi}{2}\right)\left(\Gamma\left(|m-\alpha|+\frac{\xi}{2}\right)\right)^{2}}{\Gamma\left(|m-\alpha|+\frac{\xi}{4}+\frac{1}{2}\right) \Gamma(2|m-\alpha|+\xi) \Gamma(|m-\alpha|) \Gamma(|m-\alpha|+1)}\right)^{2} .
\end{aligned}
$$

For $m=N, N+1$ the following bound is sufficient:

$$
\begin{aligned}
\left\|\widetilde{g}_{3}\right\|_{2}^{2} \leqslant & \left(\frac{\pi^{2}|| m-\alpha|-| m||}{4(|m-\alpha|+|m|) \Gamma(|m-\alpha|) \Gamma(|m-\alpha|+1)}\right)^{2}\left|\frac{k R}{2}\right|^{4|m-\alpha|} \times \\
& \times\left\|H_{|m-\alpha|}^{(1)}(k r)\right\|^{4}(1+\varepsilon) .
\end{aligned}
$$

Thus, for fixed $m,\left\|\widetilde{g}_{3}\right\|_{2} \rightarrow 0$ as $R \rightarrow 0$ provided we choose $\xi<2$.

To make the bound for $\left\|\widetilde{g}_{3}\right\|_{2}$ in (3.38) independent of $m$, we use the following limit:

$$
\lim _{n \rightarrow \infty} n^{b-a} \frac{\Gamma(n+a)}{\Gamma(n+b)}=1
$$


to deduce that there exists $M_{0} \in \mathbb{N}$ such that, if $|m|>M_{0}$, then

$$
\frac{\left(\Gamma\left(|m-\alpha|+\frac{\xi}{2}+\frac{1}{2}\right) \Gamma(2|m-\alpha|+\xi / 2)\right)^{2}(\Gamma(|m-\alpha|+\xi / 2))^{4}}{\left(\Gamma\left(|m-\alpha|+\frac{\xi}{4}+\frac{1}{2}\right) \Gamma(2|m-\alpha|+\xi) \Gamma(|m-\alpha|) \Gamma(|m-\alpha|+1)\right)^{2}} \leqslant 2^{-\xi} \bar{m}^{\frac{3}{2} \xi-2}
$$

where $\bar{m}=\lfloor|m-\alpha|\rfloor$, and we must choose $\xi<\frac{4}{3}$.

Similarly, for $m \neq N, N+1$,

$$
\begin{aligned}
\left\|\widetilde{g}_{4}\right\|_{2}^{2}= & \int\left|\widetilde{g}_{4}\left(r, r^{\prime}\right)\right|^{2} r d r r^{\prime} d r^{\prime} \\
= & \frac{\pi^{2}}{2\left|A_{m, R}^{(1)}(k)\right|^{2}} \int_{0}^{R} r d r\left|J_{|m|}(k r)\right|^{2} \int_{R}^{\infty} r^{\prime} d r^{\prime}\left|H_{|m-\alpha|}^{(1)}\left(k r^{\prime}\right)\right|^{2} \\
\leqslant & \frac{2^{\xi-1} R^{4-\xi}}{(|m|+1)(|m-\alpha|+|m|)^{2}}|k|^{-\xi} \frac{\Gamma\left(\frac{\xi}{2}\right) \Gamma\left(\frac{1}{2}\right)}{\Gamma\left(\frac{\xi}{4}+\frac{1}{2}\right)}(1+\varepsilon) \times \\
& \times \frac{\Gamma\left(|m-\alpha|+\frac{\xi}{2}+\frac{1}{2}\right) \Gamma\left(2|m-\alpha|+\frac{\xi}{2}\right)\left(\Gamma\left(|m-\alpha|+\frac{\xi}{2}\right)\right)^{2}}{\Gamma\left(|m-\alpha|+\frac{\xi}{4}+\frac{1}{2}\right) \Gamma(2|m-\alpha|+\xi)(\Gamma(|m-\alpha|))^{2}},
\end{aligned}
$$

while for $m=N, N+1$,

$$
\left\|\widetilde{g}_{4}\right\|_{2}^{2} \leqslant \frac{\pi^{2}\left|\frac{k}{2}\right|^{2|m-\alpha|} R^{2|m-\alpha|+2}}{(|m|+1)(|m-\alpha|+|m|)^{2}(\Gamma(|m-\alpha|))^{2}}\left\|H_{|m-\alpha|}^{(1)}(k r)\right\|^{2}(1+\varepsilon) .
$$

As previously, for fixed $m,\left\|\widetilde{g}_{4}\right\|_{2} \rightarrow 0$ as $R \rightarrow 0$ if we choose $\xi<2$.

To obtain a bound independent of $m$ in (3.44), we again use the limit in (3.40) to show that there exists $M_{1} \in \mathbb{N}$ such that, if $|m|>M_{1}$, then

$$
\frac{\Gamma\left(|m-\alpha|+\frac{\xi}{2}+\frac{1}{2}\right) \Gamma\left(2|m-\alpha|+\frac{\xi}{2}\right)\left(\Gamma\left(|m-\alpha|+\frac{\xi}{2}\right)\right)^{2}}{(1+|m|) \Gamma\left(|m-\alpha|+\frac{\xi}{4}+\frac{1}{2}\right) \Gamma(2|m-\alpha|+\xi)(\Gamma(|m-\alpha|))^{2}} \leqslant 2^{\frac{\xi}{4}} M_{1}^{\frac{3}{4} \xi-1} .
$$

Finally,

$$
\widetilde{g}_{5}\left(r, r^{\prime}\right)-g_{k, m}\left(r, r^{\prime}\right)=\frac{i \pi}{2} J_{|m-\alpha|}\left(k r_{<}\right) H_{|m-\alpha|}^{(1)}\left(k r_{>}\right) \mathbf{1}_{\mathbb{R}^{2} \backslash(R, \infty) \times(R, \infty)}
$$

SO

$$
\begin{aligned}
\left\|\widetilde{g}_{5}-g_{k, m}\right\|_{2}^{2}= & \frac{\pi^{2}}{2} \int_{0}^{R} r d r\left|J_{|m-\alpha|}(k r)\right|^{2} \int_{r}^{R} r^{\prime} d r^{\prime}\left|H_{|m-\alpha|}^{(1)}\left(k r^{\prime}\right)\right|^{2}+ \\
& +\frac{\pi^{2}}{2} \int_{0}^{R} r d r\left|J_{|m-\alpha|}(k r)\right|^{2} \int_{R}^{\infty} r^{\prime} d r^{\prime}\left|H_{|m-\alpha|}^{(1)}\left(k r^{\prime}\right)\right|^{2} .
\end{aligned}
$$

The first term is bounded by $\frac{R^{4}(1+\varepsilon)}{8|m-\alpha|^{2}(1+|m-\alpha|)}$, while for the second term we need to consider the cases $m \neq N, N+1$ separately using equation (3.37). 
Then we deduce that for $m \neq N, N+1$,

$$
\begin{aligned}
\| \widetilde{g}_{5}- & g_{k, m} \|_{2}^{2} \leqslant \frac{R^{4}(1+\varepsilon)}{8|m-\alpha|^{2}(1+|m-\alpha|)}+\frac{\left(\frac{2}{k}\right)^{\xi} \Gamma\left(\frac{1}{2}\right) R^{4-\xi}}{8(1+|m-\alpha|)}(1+\varepsilon) \times \\
& \times \frac{\Gamma\left(\frac{\xi}{2}\right) \Gamma\left(|m-\alpha|+\frac{\xi}{2}+\frac{1}{2}\right) \Gamma(2|m-\alpha|+\xi / 2)(\Gamma(|m-\alpha|+\xi / 2))^{2}}{\Gamma\left(\frac{\xi}{4}+\frac{1}{2}\right) \Gamma\left(|m-\alpha|+\frac{\xi}{4}+\frac{1}{2}\right) \Gamma(2|m-\alpha|+\xi)(\Gamma(|m-\alpha|+1))^{2}},
\end{aligned}
$$

while for $m=N, N+1$,

$$
\begin{aligned}
\left\|\widetilde{g}_{5}-g_{k, m}\right\|_{2}^{2} \leqslant & \frac{R^{4}(1+\varepsilon)}{8|m-\alpha|^{2}(1+|m-\alpha|)}+ \\
& +\frac{\pi^{2}\left|\frac{k}{2}\right|^{2|m-\alpha|} R^{2|m-\alpha|+2}}{4(1+|m-\alpha|)(\Gamma(1+|m-\alpha|))^{2}}\left\|H_{|m-\alpha|}^{(1)}(k r)\right\|^{2}(1+\varepsilon) .
\end{aligned}
$$

A similar argument to that used previously shows that the bound in (3.50) may be taken to be independent of $m$. This completes the proof of Lemma 1.

Next we shall show that the operator $h_{m, R}^{\beta}$ converges in norm the resolvent sense to the appropriate limit provided $\beta$ obeys condition I or II.

\section{Lemma 2:}

(a) If condition I (condition II) holds, then for $m \neq N(m \neq N+1)$, the operator $h_{m, R}^{\beta}$ converges to $h_{m}$ in the norm resolvent sense as $R \rightarrow 0$.

Furthermore, there exists $M \in \mathbb{N}$ such that for $|m|>M$, there exists a constant $c(R)$, independent of $m$, such that $\left\|g_{k, m, R}^{\beta}-g_{k, m}\right\|_{2} \leqslant c(R)$, and $\lim _{R \rightarrow 0} c(R)=0$.

(b) If condition I (condition II) holds, then the operator $h_{N, R}^{\beta}$ converges to $h_{N, \nu_{N}}\left(h_{N+1, R}^{\beta}\right.$ converges to $h_{N+1, \nu_{N+1}}$ ) in the norm resolvent sense as $R \rightarrow 0$.

Proof: We shall prove the Lemma for the case when condition I holds. The corresponding proof for the case when condition II holds is similar.

First we note the limiting behaviour of $g_{k, m, R}^{0}(R, R)$ for small $R$ :

$$
g_{k, m, R}^{0}(R, R) \simeq \begin{cases}\frac{1}{|m-\alpha|+|m|}\left(1-\tilde{c}^{\prime}(k) R^{2|m-\alpha|}\right) & m=N, N+1, \\ \frac{1}{|m-\alpha|+|m|}\left(1-d(k) R^{2}\right) & m \neq N, N+1 .\end{cases}
$$

where

$$
\tilde{c}^{\prime}(k)=\frac{2 e^{-i \pi|m-\alpha|} \Gamma(1-|m-\alpha|)}{(|m-\alpha|+|m|) \Gamma(|m-\alpha|)}\left(\frac{k}{2}\right)^{2|m-\alpha|}
$$

and

$$
d(k)=\frac{1}{1-|m-\alpha|}\left(1+\frac{|m-\alpha|-|m|}{|m-\alpha|(|m-\alpha|+|m|)}\right)\left(\frac{k}{2}\right)^{2} .
$$


(a) Let $m \neq N$ and suppose condition I holds. We need to show that

$$
\lim _{R \rightarrow 0}\left\|g_{k, m, R}^{\beta}-g_{k, m}\right\|_{2}=0
$$

Now

$$
\begin{aligned}
\left\|g_{k, m, R}^{\beta}-g_{k, m}\right\|_{2} \leqslant & \left\|g_{k, m, R}^{0}-g_{k, m}\right\|_{2}+ \\
& +\left|\frac{\beta}{1+\beta g_{k, m, R}^{0}(R, R)}\right|\left\|g_{k, m, R}^{0}(\cdot, R)\right\|^{2} .
\end{aligned}
$$

In Lemma 1, we have shown that $\lim _{R \rightarrow 0}\left\|g_{k, m, R}^{0}-g_{k, m}\right\|_{2}=0$.

The following three cases need to be considered separately:

Case 1. $m=0,1,2, \ldots, N-1$;

Case 2. $m=N+1$.

Case $3 . m \leqslant-1$ or $m \geqslant N+2$.

Case 1 . If $m=0,1,2, \ldots, N-1$, then $|m-\alpha|+|m|=\alpha$. For small R,

$$
\left|\frac{\beta}{1+\beta g_{k, m, R}^{0}(R, R)}\right| \simeq \frac{\alpha^{2}}{2 \delta\left|\nu_{N}\right|} R^{-2 \delta},
$$

while

$$
\begin{aligned}
\left\|g_{k, m, R}^{0}(\cdot, R)\right\|^{2}= & \int_{0}^{\infty} r d r\left|g_{k, m, R}^{0}(r, R)\right|^{2} \\
= & \frac{\pi^{2}}{4\left|A_{m, R}^{(1)}(k)\right|^{2}}\left\{\left|H_{|m-\alpha|}^{(1)}(k R)\right|^{2} \int_{0}^{R} r d r\left|J_{|m|}(k r)\right|^{2}\right. \\
& \left.\quad+\left|J_{|m|}(k R)\right|^{2} \int_{R}^{\infty} r d r\left|H_{|m-\alpha|}^{(1)}(k r)\right|^{2}\right\} \\
\leqslant & (1+\varepsilon)\left[\frac{R^{2}}{2 \alpha^{2}(1+|m|)}+\frac{R^{2-\xi}}{2 \alpha^{2}}\left(\frac{2}{k}\right)^{\xi} \frac{\Gamma\left(\frac{\xi}{2}\right) \Gamma\left(\frac{1}{2}\right)}{\Gamma\left(\frac{\xi}{4}+\frac{1}{2}\right)} \times\right. \\
& \left.\times \frac{\Gamma\left(|m-\alpha|+\frac{\xi+1}{2}\right) \Gamma(2|m-\alpha|+\xi / 2)(\Gamma(|m-\alpha|+\xi / 2))^{2}}{\Gamma\left(|m-\alpha|+\frac{\xi+2}{4}\right) \Gamma(2|m-\alpha|+\xi)(\Gamma(|m-\alpha|))^{2}}\right]
\end{aligned}
$$

Taking $\xi<2(1-\delta)$ gives the desired limit.

Case 2. If $m=N+1$, then $|m-\alpha|+|m|=N+2-\delta$. Then

$$
\lim _{R \rightarrow 0}\left|\frac{\beta}{1+\beta g_{k, N+1, R}^{0}(R, R)}\right|=\frac{\alpha(N+2-\delta)}{2(1-\delta)},
$$


while

$$
\begin{aligned}
\int_{0}^{\infty} r d r\left|g_{k, N+1, R}^{0}(r, R)\right|^{2}= & \frac{\pi^{2}}{4\left|A_{N+1, R}^{(1)}(k)\right|^{2}}\left\{\left|H_{1-\delta}^{(1)}(k R)\right|^{2} \int_{0}^{R} r d r\left|J_{N+1}(k r)\right|^{2}+\right. \\
& \left.+\left|J_{N+1}(k R)\right|^{2} \int_{R}^{\infty} r d r\left|H_{1-\delta}^{(1)}(k r)\right|^{2}\right\} \\
\leqslant & \frac{R^{2}}{2(N+2)(N+2-\delta)^{2}}(1+\varepsilon)+ \\
& +\frac{\pi^{2}\left\|H_{1-\delta}^{(1)}(k r)\right\|^{2}}{(N+2-\delta)^{2}(\Gamma(1-\delta))^{2}}\left|\frac{k R}{2}\right|^{2(1-\delta)}(1+\varepsilon) .
\end{aligned}
$$

Case 3. If $m \leqslant-1$ or $m \geqslant N+2$, then $|m-\alpha|+|m|=|2 m-\alpha|$. The constant term is bounded as follows:

$$
\left|\frac{\beta}{1+\beta g_{k, m, R}^{0}(R, R)}\right| \leqslant \frac{\alpha|2 m-\alpha|}{|2 m-\alpha|-\alpha}(1+\varepsilon),
$$

and

$$
\begin{aligned}
& \int_{0}^{\infty} r d r\left|g_{k, m, R}^{0}(r, R)\right|^{2}=\frac{\pi^{2}}{4\left|A_{m, R}^{(1)}(k)\right|^{2}}\left\{\left|H_{|m-\alpha|}^{(1)}(k R)\right|^{2} \int_{0}^{R} r d r\left|J_{|m|}(k r)\right|^{2}+\right. \\
&\left.+\left|J_{|m|}(k R)\right|^{2} \int_{R}^{\infty} r d r\left|H_{|m-\alpha|}^{(1)}(k r)\right|^{2}\right\} \\
& \leqslant {\left[\frac{R^{2}}{2|2 m-\alpha|^{2}(1+|m|)}+\frac{R^{2-\xi}}{2|2 m-\alpha|^{2}}\left(\frac{2}{k}\right)^{\xi} \frac{\Gamma\left(\frac{\xi}{2}\right) \Gamma\left(\frac{1}{2}\right)}{\Gamma\left(\frac{\xi}{4}+\frac{1}{2}\right)}(1+\varepsilon) \times\right.} \\
&\left.\times \frac{\Gamma\left(|m-\alpha|+\frac{\xi+1}{2}\right) \Gamma(2|m-\alpha|+\xi / 2)(\Gamma(|m-\alpha|+\xi / 2))^{2}}{\Gamma\left(|m-\alpha|+\frac{\xi+2}{2}\right) \Gamma(2|m-\alpha|+\xi)(\Gamma(|m-\alpha|))^{2}}\right],
\end{aligned}
$$

An argument similar to that used in (3.46) ensures that the bound is independent of $m$ if $|m|$ is large enough.

(b) Let $m=N$ and suppose condition I holds. Then

$$
\left\|g_{k, N, R}^{\beta}-g_{k, N}^{\nu_{N}}\right\|_{2} \leqslant\left\|g_{k, N, R}^{0}-g_{k, N}\right\|_{2}+\left\|l_{k, N, R}-l_{k, N}\right\|_{2}
$$

where

$$
l_{k, N, R}\left(r, r^{\prime}\right)=\frac{\beta}{1+\beta g_{k, N, R}^{0}(R, R)} g_{k, N, R}^{0}(r, R) g_{k, N, R}^{0}\left(R, r^{\prime}\right),
$$

and

$$
l_{k, N}=c\left(N, \nu_{N}, k\right) H_{\delta}^{(1)}(k r) H_{\delta}^{(1)}\left(k r^{\prime}\right) .
$$

As noted previously, Lemma 1 proves that $\lim _{R \rightarrow 0}\left\|g_{k, N, R}^{\beta}-g_{k, N}^{\nu_{N}}\right\|_{2}=0$. 
Now,

$$
\begin{aligned}
\left\|l_{k, N, R}-l_{k, N}\right\|_{2}^{2}= & \int r d r r^{\prime} d r^{\prime} \mid \frac{\beta}{1+\beta g_{k, N, R}^{0}(R, R)} g_{k, N, R}^{0}(r, R) g_{k, N, R}^{0}\left(R, r^{\prime}\right)- \\
& -\left.c\left(N, \nu_{N}, k\right) H_{\delta}^{(1)}(k r) H_{\delta}^{(1)}\left(k r^{\prime}\right)\right|^{2} \\
= & l^{(1)}+l^{(2)}-l^{(3)}-\overline{l^{(3)}}
\end{aligned}
$$

where

$$
\begin{aligned}
& l^{(1)}=\left|\frac{\beta}{1+\beta g_{k, N, R}^{0}(R, R)}\right|^{2} \int r d r r^{\prime} d r^{\prime}\left|g_{k, N, R}^{0}(r, R) g_{k, N, R}^{0}\left(R, r^{\prime}\right)\right|^{2}, \\
& l^{(2)}=\left|c\left(N, \nu_{N}, k\right)\right|^{2} \int r d r r^{\prime} d r^{\prime}\left|H_{\delta}^{(1)}(k r) H_{\delta}^{(1)}\left(k r^{\prime}\right)\right|^{2},
\end{aligned}
$$

and

$$
\begin{aligned}
l^{(3)}= & \frac{\beta}{1+\beta g_{k, N, R}^{0}(R, R)} \overline{c\left(N, \nu_{N}, k\right)} \int r d r r^{\prime} d r^{\prime} g_{k, N, R}^{0}(r, R) \times \\
& \times g_{k, N, R}^{0}\left(R, r^{\prime}\right) \overline{H_{\delta}^{(1)}(k r) H_{\delta}^{(1)}\left(k r^{\prime}\right)}
\end{aligned}
$$

For small $R$,

$$
\frac{\beta}{1+\beta g_{k, N, R}^{0}(R, R)} \simeq \frac{-\alpha^{2} R^{-2 \delta}}{2 \delta \nu_{N}+\alpha \tilde{c}^{\prime}(k)}
$$

Then

$$
\begin{aligned}
& \int r d r r^{\prime} d r^{\prime}\left|g_{k, N, R}^{0}(r, R) g_{k, N, R}^{0}\left(R, r^{\prime}\right)\right|^{2}=\frac{\pi^{4}}{16\left|A_{N, R}^{(1)}(k)\right|^{4}} \times \\
& \quad \times\left\{\left|H_{\delta}^{(1)}(k R)\right|^{4}\left[\int_{0}^{R} r d r\left|J_{N}(k r)\right|^{2}\right]^{2}+\left|J_{N}(k R)\right|^{4}\left[\int_{R}^{\infty} r d r\left|H_{\delta}^{(1)}(k R)\right|^{2}\right]^{2}\right\}
\end{aligned}
$$

The first term is bounded by $\frac{R^{4}}{4 \alpha^{4}(N+1)^{2}}(1+\varepsilon)$, while for small $R$

$$
\begin{aligned}
& \left|\frac{\beta}{1+\beta g_{k, N, R}^{0}(R, R)}\right|^{2} \frac{\pi^{4}\left|J_{N}(k R)\right|^{4}}{16\left|A_{N, R}^{(1)}(k)\right|^{4}}\left[\int_{R}^{\infty} r d r\left|H_{\delta}^{(1)}(k R)\right|^{2}\right]^{2} \simeq \\
& \quad \frac{\pi^{4}\left(\frac{k}{2}\right)^{4 \delta}}{(\Gamma(\delta))^{4}\left(2 \delta \nu_{N}+\alpha \tilde{c}^{\prime}(k)\right)^{2}}\left\|H_{\delta}^{(1)}(k r) \mathbf{1}_{(R, \infty)}\right\|^{4}=\left|c\left(N, \nu_{N}, k\right)\right|^{2}\left\|H_{\delta}^{(1)}(k r) \mathbf{1}_{(R, \infty)}\right\|^{4} .
\end{aligned}
$$

Then $l^{(1)} \rightarrow l^{(2)}$ as $R \rightarrow 0$ by dominated convergence. 
For $l^{(3)}$ we have

$$
\begin{aligned}
\int r d r r^{\prime} d r^{\prime} g_{k, N, R}^{0}(r, R) & g_{k, N, R}^{0}\left(R, r^{\prime}\right) \overline{H_{\delta}^{(1)}(k r) H_{\delta}^{(1)}\left(k r^{\prime}\right)}= \\
= & {\left[\frac { i \pi } { 2 A _ { N , R } ^ { ( 1 ) } ( k ) } \left\{H_{\delta}^{(1)}(k R) \int_{0}^{R} r d r J_{N}(k r) \overline{H_{\delta}^{(1)}(k r)}+\right.\right.} \\
& \left.\left.+J_{N}(k R) \int_{R}^{\infty} r d r\left|H_{\delta}^{(1)}(k r)\right|^{2}\right\}\right]^{2} .
\end{aligned}
$$

The first term in the bracket goes to $\frac{i \Gamma(\delta) R^{2-\delta}}{\alpha \pi(N+2-\delta)}\left(\frac{\bar{k}}{2}\right)^{-\delta}$, while for small $R$,

$$
\frac{i \pi}{2 A_{N, R}^{(1)}(k)} J_{N}(k R) \int_{R}^{\infty} r d r\left|H_{\delta}^{(1)}(k r)\right|^{2} \simeq \frac{i \pi}{\alpha \Gamma(\delta)}\left|\frac{k R}{2}\right|^{\delta}\left\|H_{\delta}^{(1)}(k r) \mathbf{1}_{(R, \infty)}\right\|^{2} .
$$

Then $l^{(3)} \rightarrow l^{(2)}$ as $R \rightarrow 0$ by dominated convergence, which completes the proof.

\section{Approximation by Smooth Flux Tube}

In Section 3, $\mathbf{A}_{R}=\alpha \frac{\mathbf{k} \times \mathbf{r}}{r^{2}} \Theta(r-R)$ so that $\mathbf{B}_{R}$ is concentrated on a cylindrical shell. Now we replace the $\Theta$-function by a smooth step function which approximates the $\Theta$-function as $R \rightarrow 0$. Let $a: \mathbb{R} \mapsto \mathbb{R}$ be a differentiable function with $a(r)=0$ for $r \leqslant 0$ and $a(r)=1$ for $r \geqslant r_{0}$, where $r_{0}>0$. Furthermore, let $a$ be such that the function $b(r)=\frac{1}{r} \frac{d}{d r} a(r)$ is bounded in absolute value (i.e. there exists $b_{0} \in \mathbb{R}$ such that $\left.|b(r)| \leqslant b_{0}\right)$. Then $b(r)$ has support only in $\left(0, r_{0}\right)$, and $\int_{0}^{\infty} b(r) r d r=1$. We take

$$
\hat{\mathbf{A}}_{R}=\alpha \frac{\mathbf{k} \times \mathbf{r}}{r^{2}} a\left(\frac{r-R}{R^{5}}\right)
$$

so that $\mathbf{k} \cdot \hat{\mathbf{B}}_{R}=R^{-5} \alpha b\left(\frac{r-R}{R^{5}}\right)$. Let

$$
\widehat{H}_{R}=\left(i \nabla+\hat{\mathbf{A}}_{R}\right)^{2}+\frac{\beta}{\alpha} \mathbf{k} \cdot \hat{\mathbf{B}}_{R} .
$$

$\beta$ here depends on $\alpha$ and $R$. Motivated by the result of Section 3 we shall consider two cases:

(a) $\beta(\alpha, R) \simeq-\alpha\left(1-\frac{2 \delta}{\alpha} \nu_{N} R^{2 \delta}\right)$,

(b) $\beta(\alpha, R) \simeq(\alpha-2(N+1))\left(1-\frac{2(1-\delta)}{2(N+1)-\alpha} \nu_{N+1} R^{2(1-\delta)}\right)$.

The component of the operator $\widehat{H}_{R}$ on the space with angular momentum $m$ are

$$
\hat{h}_{m, R}=-\frac{1}{r} \frac{\partial}{\partial r} r \frac{\partial}{\partial r}+\frac{\left(m-\alpha a\left(\frac{r-R}{R^{5}}\right)\right)^{2}}{r^{2}}+\beta R^{-5} b\left(\frac{r-R}{R^{5}}\right) .
$$


We shall prove convergence in the norm resolvent sense of the operators $\hat{h}_{m, R}$, and hence of $\widehat{H}_{R}$.

We can rewrite the last equation as

$$
\hat{h}_{m, R}=-\frac{1}{r} \frac{\partial}{\partial r} r \frac{\partial}{\partial r}+\frac{(m-\alpha \Theta(r-R))^{2}}{r^{2}}+\frac{\beta}{R^{5} r} V_{m}\left(\frac{r-R}{R^{5}}\right)
$$

where

$$
V_{m}(r)=r b(r)+\frac{R^{4}}{\beta\left(r R^{4}+1\right)}\left\{-2 m \alpha(a(r)-\Theta(r))+\alpha^{2}\left(a^{2}(r)-\Theta(r)\right)\right\} .
$$

We note that $V_{m}(r)$ has support only in $\left(0, r_{0}\right)$, and that

$$
\left|\hat{a}_{m}(r)\right|:=\left|-2 m \alpha(a(r)-\Theta(r))+\alpha^{2}\left(a^{2}(r)-\Theta(r)\right)\right| \leqslant m K
$$

where $K$ is a constant independent of $m$ and $R$.

Define the auxiliary operators

$$
\tilde{h}_{m, R}=-\frac{1}{r} \frac{\partial}{\partial r} r \frac{\partial}{\partial r}+\frac{\left(m-\alpha \Theta\left(r-\frac{1}{R^{4}}\right)\right)^{2}}{r^{2}}+\frac{R^{5} \beta}{r} V_{m}\left(r-\frac{1}{R^{4}}\right) .
$$

$V_{m}$ is form compact (Note 4, Appendix 1) with respect to

$$
-\frac{1}{r} \frac{\partial}{\partial r} r \frac{\partial}{\partial r}+\frac{(m-\alpha \Theta(r-R))^{2}}{r^{2}}
$$

so the form sum (4.7) is well-defined. From Thm. B.1(b) of [7], the resolvent of $\tilde{h}_{m, R}$ for $k^{2} \in \rho\left(\tilde{h}_{m, R}\right)$ and $\Im(k)>0$, is given by

$$
\left[\tilde{h}_{m, R}-k^{2}\right]^{-1}=g_{k, m, R}^{0}-R^{5} \beta g_{k, m, R}^{0} \tilde{v}_{m}\left[1+\beta \tilde{B}_{m, R}(k)\right]^{-1} \tilde{u}_{m} g_{k, m, R}^{0}
$$

where

$$
\tilde{v}_{m}(r)=\left|\frac{1}{r} V_{m}\left(r-\frac{1}{R^{4}}\right)\right|^{\frac{1}{2}}, \quad \tilde{u}_{m}(r)=\left|\frac{1}{r} V_{m}\left(r-\frac{1}{R^{4}}\right)\right|^{\frac{1}{2}} \operatorname{sgn}\left[V_{m}\left(r-\frac{1}{R^{4}}\right)\right]
$$

and

$$
\tilde{B}_{m, R}(k)=R^{5} \tilde{u}_{m} g_{k, m, R}^{0} \tilde{v}_{m} ; \quad \Im(k)>0 .
$$

$\tilde{B}_{m, R}$ extends to a Hilbert-Schmidt operator (Note 4, Appendix 1).

Introducing the unitary scaling group $\left(U_{R} g\right)(r)=\frac{1}{R^{5}} g\left(\frac{r}{R^{5}}\right)$, we get

$$
\hat{h}_{m, R}=\frac{1}{R^{10}} U_{R} \tilde{h}_{m, R} U_{R}^{-1} .
$$

Then, noting that

$$
R^{10} U_{R} g_{k, m, R}^{0} U_{R}^{-1}=g_{k / R^{5}, m, R}^{0}
$$


and taking the translation $r \rightarrow r+\frac{1}{R^{4}}$, we obtain

$$
\begin{aligned}
{\left[\hat{h}_{m, R}-k^{2}\right]^{-1}=} & {\left[\frac{1}{R^{10}} U_{R} \tilde{h}_{m, R} U_{R}^{-1}-k^{2}\right]^{-1} } \\
= & R^{10} U_{R}\left[\tilde{h}_{m, R}-\left(R^{5} k\right)^{2}\right]^{-1} U_{R}^{-1} \\
= & R^{10} U_{R}\left[g_{R^{5} k, m, R}^{0}-\right. \\
& \left.-R^{5} \beta g_{R^{5} k, m, R}^{0} \tilde{v}_{m}\left[1+\beta \tilde{B}_{m, R}\left(R^{5} k\right)\right]^{-1} \tilde{u}_{m} g_{R^{5} k, m, R}^{0}\right] U_{R}^{-1}
\end{aligned}
$$

for $k^{2} \in \rho(\hat{h})$ and $\Im(k)>0$. For $\Im(k)>0$, define Hilbert-Schmidt operators $A_{m, R}(k)$, $B_{m, R}(k)$ and $C_{m, R}(k)$, with integral kernels:

$$
\begin{aligned}
& A_{m, R}\left(k, r, r^{\prime}\right)=g_{k, m, R}^{0}\left(r, R\left(1+R^{4} r^{\prime}\right)\right) \hat{v}_{m}\left(r^{\prime}\right) \\
& B_{m, R}\left(k, r, r^{\prime}\right)=\hat{u}_{m}(r) g_{k, m, R}^{0}\left(R\left(1+R^{4} r\right), R\left(1+R^{4} r^{\prime}\right)\right) \hat{v}_{m}\left(r^{\prime}\right) \\
& C_{m, R}\left(k, r, r^{\prime}\right)=\hat{u}_{m}(r) g_{k, m, R}^{0}\left(R\left(1+R^{4} r\right), r^{\prime}\right)
\end{aligned}
$$

where

$$
\hat{v}_{m}(r)=\left|\frac{V_{m}(r)}{r}\right|^{\frac{1}{2}} \quad \text { and } \quad \hat{u}_{m}(r)=\left|\frac{V_{m}(r)}{r}\right|^{\frac{1}{2}} \operatorname{sgn}\left[V_{m}(r)\right] .
$$

Then (4.13) becomes

$$
\left[\hat{h}_{m, R}-k^{2}\right]^{-1}=g_{k, m, R}^{0}-\beta A_{m, R}(k)\left[1+\beta B_{m, R}(k)\right]^{-1} C_{m, R}(k)
$$

for $k^{2} \in \rho\left(h_{\varepsilon, m, R}\right)$ and $\Im(k)>0$.

Using this representation we can prove the following result.

\section{Theorem 2.}

Let

$$
\widehat{H}_{R}=\bigoplus_{m=-\infty}^{\infty} \hat{h}_{m, R}
$$

Then $\widehat{H}_{R}$ converges, as $R \rightarrow 0$, to one of the self-adjoint extensions $H^{\boldsymbol{\nu}}$ of the A-B Hamiltonian only if either

$$
\begin{aligned}
& \text { (I) } \frac{\beta(\alpha, R)+\alpha}{R^{2 \delta}} \rightarrow 2 \delta \nu_{N} \\
& \text { or }{ }_{\text {(II) }} \frac{\beta(\alpha, R)-\alpha+2(N+1)}{R^{2(1-\delta)}} \rightarrow 2(1-\delta) \nu_{N+1} \text {. }
\end{aligned}
$$

In case (I) $\widehat{H}_{R}$ converges in the norm resolvent sense, as $R \rightarrow 0$, to $H^{\left(\nu_{N}, \infty\right)}$, and in case (II) to $H^{\left(\infty, \nu_{N+1}\right)}$.

The proof of this theorem is fairly standard but by no means trivial. Because again we require uniform convergence in $m$ we need to control the $m$-behaviour and this makes the proof very lengthy. We therefore we do not give the proof here but only state the two lemmas required in the case when Condition I holds. Once we have these two lemmas, the proof is similar to that of Theorem 1. and the result follows from them. 
We have already proved that $g_{k, m, R}^{0} \rightarrow g_{k, m}$ in norm. Let

$$
v(r)=|b(r)|^{\frac{1}{2}} ; \quad u(r)=|b(r)|^{\frac{1}{2}} \operatorname{sgn}[b(r)] .
$$

Lemma 3: If condition I holds, then

(a) for $m<0$ and $m>N$, the operators $A_{m, R}(k), C_{m, R}(k) \rightarrow 0$ in norm;

(b) for $m=0, \ldots, N-1$, the operators $R^{-\delta} A_{m, R}(k), R^{-\delta} C_{m, R}(k) \rightarrow 0$ in norm;

(c) for $m=N, R^{-\delta} A_{m, R}(k) \rightarrow A_{N}(k)$ and $R^{-\delta} C_{m, R}(k) \rightarrow C_{N}(k)$ in norm, where $A_{N}\left(k, r, r^{\prime}\right)=\tilde{c}_{N}(k) H_{\delta}^{(1)}(k r) v\left(r^{\prime}\right)$ and $C_{N}\left(k, r, r^{\prime}\right)=\tilde{c}_{N}(k) u(r) H_{\delta}^{(1)}\left(k r^{\prime}\right)$.

\section{Lemma 4:}

If condition I holds, then

(a) for $m<0$ and $m>N,\left\|\beta(\alpha, R) B_{m, R}(k)\right\|_{2} \leqslant \frac{C|\alpha|}{|m-\alpha|+|m|}$, where $C$ is a constant independent of $m$ and $R$;

(b) for $m=0, \ldots, N, R^{2 \delta}\left[1+\beta(\alpha, R) B_{m, R}\right]^{-1} \rightarrow \frac{1}{\kappa_{m}}\langle v, \cdot\rangle u$ in norm as $R \rightarrow 0$, where

$$
\kappa_{m}= \begin{cases}-\frac{\alpha}{2 \delta \nu_{N}} & m=0, \ldots, N-1 \\ \frac{\alpha \tilde{c}_{N}^{2}(k)}{c\left(N, \nu_{N}, k\right)} & m=N\end{cases}
$$

\section{Other Approximations}

As mentioned in the Introduction, there are two other very natural approximations. These were investigated also in $[3,4]$. Case $(1)$ is when the magnetic field inside the cylinder of radius $R$ is homogeneous, that is,

$$
\mathbf{A}_{R}= \begin{cases}\alpha \frac{\mathbf{k} \times \mathbf{r}}{R^{2}}, & r<R \\ \alpha \frac{\mathbf{k} \times \mathbf{r}}{r^{2}}, & r>R\end{cases}
$$

Here $\mathbf{k} \cdot \mathbf{B}_{R}=\frac{2 \alpha}{R^{2}} \Theta(R-r)$.

Case (2) is when the magnetic field is proportional to $\frac{1}{r}$ inside the cylinder:

$$
\mathbf{A}_{R}= \begin{cases}\alpha \frac{\mathbf{k} \times \mathbf{r}}{r R}, & r<R \\ \alpha \frac{\mathbf{k} \times \mathbf{r}}{r^{2}}, & r>R .\end{cases}
$$


In this case $\mathbf{k} \cdot \mathbf{B}_{R}=\frac{\alpha}{r R} \Theta(R-r)$.

Let

$$
\sigma= \begin{cases}\frac{1+N}{M(1,2+N, \alpha)}, & \text { in case (1) } \\ \frac{1+2 N}{M(1,2+2 N, 2 \alpha)}, & \text { in case (2), }\end{cases}
$$

where $M(a, b, z)$ is Kummer's function. Let $\eta$ be a solution of the equation

$$
\eta=-\frac{(1-\delta)(2+N) M(\eta, 2+N, \alpha)}{\alpha M(\eta+1,3+N, \alpha)},
$$

in case (1) and of the equation

$$
\eta=-\frac{(1-\delta)(3+2 N) M(\eta, 3+2 N, 2 \alpha)}{\alpha M(\eta+1,4+2 N, 2 \alpha)}
$$

in case (2). Note that both these equations have an infinite number of solutions.

Let

$$
\phi_{k, m, R}\left(r, r^{\prime}\right)=g_{k, m, R}^{\beta}\left(r, r^{\prime}\right)-g_{k, m}\left(r, r^{\prime}\right)
$$

where $g_{k, m, R}^{\beta}\left(r, r^{\prime}\right)$ is the resolvent of the approximating Hamiltonian in each case and $g_{k, m}\left(r, r^{\prime}\right)$ is as in (2.4).

The following are the only cases which give non-trivial results:

(I) if $\beta \simeq-\alpha\left(1-\sigma \frac{2 \delta}{\alpha} \nu_{N} R^{2 \delta}\right)$ as $R \rightarrow 0$, then $\phi_{k, m, R}\left(r, r^{\prime}\right)$ approaches

1. a non-zero limit for $m=N$, corresponding to the self-adjoint extension $h_{N, \nu_{N}}$;

2. zero limit for $m=N+1$, corresponding to the regular self-adjoint extension $h_{N+1, \infty}$;

3. zero limit for $m \neq N, N+1$, corresponding to the self-adjoint operators $h_{m}$.

(II) if $\beta \simeq \alpha(2 \eta-1)\left(1-\frac{2 \eta}{2 \eta-1} \nu_{N+1} R^{2(1-\delta)}\right)$ as $R \rightarrow 0$, then $\phi_{k, m, R}\left(r, r^{\prime}\right)$ approaches

1. a non-zero limit for $m=N+1$, corresponding to the self-adjoint extension $h_{N+1, \nu_{N+1}}$;

2. zero limit for $m=N$, corresponding to the regular self-adjoint extension $h_{N, \infty}$;

3. zero limit for $m \neq N, N+1$, as before.

Acknowledgement: One of the authors (J.L.B.) would like to thank the University of Malta (Staff Development Fund) for financial support during the course of this work. 


\section{Appendix 1}

Note 1:

The Bessel functions of the first and third kind have the following limiting and asymptotic properties [8]:

1. for small $z$,

$$
\begin{aligned}
J_{\nu}(z) & \simeq \frac{1}{\Gamma(\nu+1)}\left(\frac{z}{2}\right)^{\nu} \quad(\nu \neq-1,-2,-3, \ldots) \\
H_{\nu}^{(1)}(z) & \simeq \frac{-i \Gamma(\nu)}{\pi}\left(\frac{z}{2}\right)^{-\nu} \quad(\mathcal{R} \nu>0)
\end{aligned}
$$

2. for large $|z|$,

$$
\begin{aligned}
& J_{\nu}(z)=\sqrt{\frac{2}{\pi z}}\left\{\cos \left(z-\frac{\nu \pi}{2}-\frac{\pi}{4}\right)+e^{|\mathcal{I} z|} O\left(|z|^{-1}\right)\right\} \\
& (|\arg z|<\pi) \\
& H_{\nu}^{(1)}(z)=\sqrt{\frac{2}{\pi z}} e^{i\left(z-\frac{\nu \pi}{2}-\frac{\pi}{4}\right)} \quad(-\pi<\arg z<2 \pi)
\end{aligned}
$$

Two linearly independent solutions of

$$
\left(-\frac{1}{r} \frac{\partial}{\partial r} r \frac{\partial}{\partial r}+\frac{\nu^{2}}{r^{2}}\right) \phi_{k}(r)=k^{2} \phi_{k}(r)
$$

are $J_{\nu}(k r)$ and $H_{\nu}^{(1)}(k r)$.

In the given case, the only solution for $r<R$ which lies in $\mathcal{D}\left(h_{m, R}^{*}\right)$ is $J_{|m|}(k r)$, while for $r>R$ the only solution is $H_{|m-\alpha|}^{(1)}(k r)$.

\section{Note 2:}

To obtain the Green's function, consider two solutions of equation (6.5), one of which is regular at $r=0$ and irregular at $r=\infty$, while the other is irregular at $r=0$ and regular at $r=\infty$.

Consider

$$
\begin{aligned}
& \phi_{1, m, k}(r)= \begin{cases}J_{|m|}(k r) & r<R \\
A_{m}^{(1)}(k) J_{|m-\alpha|}(k r)+B_{m}^{(1)}(k) H_{|m-\alpha|}^{(1)}(k r) & r>R\end{cases} \\
& \phi_{2, m, k}(r)= \begin{cases}A_{m, R}^{(2)}(k) J_{|m|}(k r)+B_{m, R}^{(2)}(k) H_{|m|}^{(1)}(k r) & r<R \\
H_{|m-\alpha|}^{(1)}(k r) & r>R\end{cases}
\end{aligned}
$$

where the constants $A_{m, R}^{(1)}(k), A_{m, R}^{(2)}(k), B_{m, R}^{(1)}(k)$ and $B_{m, R}^{(2)}(k)$ are chosen so that the boundary conditions $\phi_{m, k}(R+)=\phi_{m, k}(R-)$ and $\phi_{m, k}^{\prime}(R+)=\phi_{m, k}^{\prime}(R-)$ are satisfied (note that the second boundary condition is the one imposed to obtain the regular self-adjoint extension i.e. the one with $\beta=0$ ). 
Then $g_{k, m, R}^{0}\left(r, r^{\prime}\right)=c \phi_{1, m, k}\left(r_{<}\right) \phi_{2, m, k}\left(r_{>}\right)$, where $r_{<}=\min \left\{r, r^{\prime}\right\}$ and $r_{>}=\max \left\{r, r^{\prime}\right\}$. The constant $c$ is determined by considering the boundary condition at $r=r^{\prime}$ :

$$
\left.\Delta \frac{\partial g_{k, m, R}^{0}}{\partial r}\right|_{r=r^{\prime}} \equiv \lim _{r \downarrow r^{\prime}} \frac{\partial g_{k, m, R}^{0}}{\partial r}\left(r, r^{\prime}\right)-\lim _{r \uparrow r^{\prime}} \frac{\partial g_{k, m, R}^{0}}{\partial r}\left(r, r^{\prime}\right)=-\frac{1}{r^{\prime}}
$$

Then we obtain

$$
g_{k, m, R}^{0}\left(r, r^{\prime}\right)= \begin{cases}\frac{i \pi}{2 B_{m, R}^{(2)}(k)} \phi_{1, m, k}\left(r_{<}\right) \phi_{2, m, k}\left(r_{>}\right) & r, r^{\prime} \leqslant R \\ \frac{i \pi}{2 A_{m, R}^{(1)}(k)} \phi_{1, m, k}\left(r_{<}\right) \phi_{2, m, k}\left(r_{>}\right) & r, r^{\prime} \geqslant R\end{cases}
$$

Note that the boundary conditions imply that

$$
\lim _{R \rightarrow 0}\left(A_{m, R}^{(1)}(k)-B_{m, R}^{(2)}(k)\right)=0 .
$$

\section{Note 3:}

(Cfr. Thm I.3.1.2 of [7]) The general structure of equation (3.14) follows from Krein's formula. To verify the constant in the second term, define for $g \in L^{2}((0, \infty), r d r)$ and $\Im(k)>0$,

$$
\begin{aligned}
f_{\beta}(r)= & \left(\left(h_{m, R}-k^{2}\right)^{-1} g\right)(r)-\frac{\beta}{1+\beta g_{k, m, R}^{0}(R, R)} \\
& \times\left\langle\overline{g_{k, m, R}^{0}(\cdot, R)}, g\right\rangle g_{k, m, R}^{0}(r, R) \\
= & \int_{0}^{\infty} d r^{\prime} r^{\prime} g_{k, m, R}^{0}\left(r, r^{\prime}\right) g\left(r^{\prime}\right)-\frac{\beta}{1+\beta g_{k, m, R}^{0}(R, R)} \\
& \times \int_{0}^{\infty} d r^{\prime} r^{\prime} g_{k, m, R}^{0}(r, R) g_{k, m, R}^{0}\left(R, r^{\prime}\right) g\left(r^{\prime}\right) .
\end{aligned}
$$

Then $f_{\beta} \in H_{l o c}^{2,2}((0, \infty) \backslash\{R\}, r d r) \cap H^{2,1}((0, \infty), r d r)$ and

$$
\begin{aligned}
f_{\beta}^{\prime}(R+)-f_{\beta}^{\prime}(R-) & =\frac{\beta / R}{1+\beta R g_{k, m, R}^{0}(R, R)} \int_{0}^{\infty} d r^{\prime} r^{\prime} g_{k, m, R}^{0}\left(R, r^{\prime}\right) g\left(r^{\prime}\right) \\
& =\frac{\beta}{R} f_{\beta}(R)
\end{aligned}
$$

This means that $f_{\beta} \in \mathcal{D}\left(h_{m, R}^{\beta}\right)$. Furthermore, for $\Im(k)>0$

$$
\left(h_{m, R}^{\beta}-k^{2}\right) f_{\beta}=g(r), \quad r \in(0, \infty) \backslash\{R\}
$$

which proves equation (3.14).

\section{Note 4:}

To show that $\tilde{B}$ extends to a Hilbert-Schmidt operator, we need to show that (cfr. [7] p.80)

$$
\int_{[0, \infty] \times[0, \infty]} d r d r^{\prime}|V(r)|\left|g_{k, m, R}^{0}\left(r, r^{\prime}\right)\right|^{2}\left|V\left(r^{\prime}\right)\right|<\infty, \quad \Im(k)>0 .
$$


This follows by considering the behaviour of the Bessel functions for small and large arguments, as discussed in Note 1. From the previous estimate we obtain

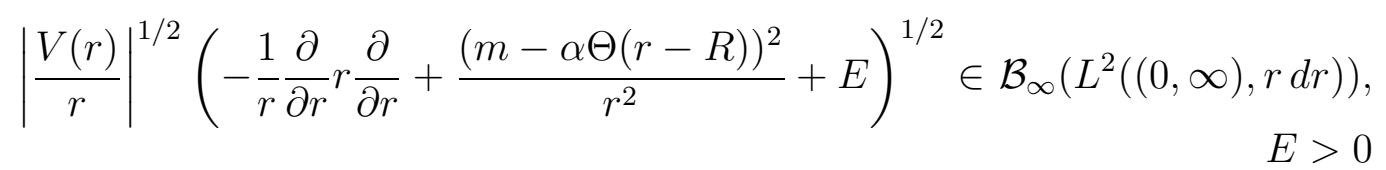

\section{Appendix 2}

In this section we shall derive bounds for expressions involving the Bessel functions $J_{\nu}(z)$ and $H_{\nu}^{(1)}(z)$.

First we shall obtain upper bounds for $\left|J_{\nu}(z)\right|$. If $\nu \geqslant-\frac{1}{2}$, then it follows [10] from Poisson's integral that

$$
\left|J_{\nu}(z)\right| \leqslant \frac{\left|\frac{z}{2}\right|^{\nu}}{\Gamma(\nu+1)} e^{|\Im z|}
$$

For $\nu<-\frac{1}{2}$, the following series expansion [8], which is valid for all $z$ and $\nu$, is used:

$$
\begin{aligned}
J_{\nu}(z) & =\left(\frac{z}{2}\right)^{\nu} \sum_{k=0}^{\infty} \frac{\left(-\frac{1}{4} z^{2}\right)^{k}}{k ! \Gamma(\nu+k+1)} \\
& =\frac{\left(\frac{z}{2}\right)^{\nu}}{\Gamma(\nu+1)}\left(1+\sum_{k=1}^{\infty} \frac{\Gamma(\nu+1)\left(-\frac{1}{4} z^{2}\right)^{k}}{k ! \Gamma(\nu+k+1)}\right) .
\end{aligned}
$$

For $\nu \neq-1,-2,-3, \ldots, \frac{|\Gamma(\nu+1)|}{|\Gamma(\nu+k+1)|} \leqslant \frac{1}{\left|\nu_{0}+1\right|^{k}}$, where $\left|\nu_{0}+1\right|=\min \{|\nu+1|,|\nu+2|, \mid \nu+$ $3 \mid, \ldots\}$. Thus we obtain:

$$
\left|J_{\nu}(z)\right| \leqslant \frac{\left|\frac{z}{2}\right|^{\nu}}{|\Gamma(\nu+1)|^{\frac{1}{4}|z|^{2}}} e^{\frac{\nu_{0}+1 \mid}{\nu_{0}}}, \quad \quad \nu \neq-1,-2,-3, \ldots
$$

Next we obtain lower bounds for $\left|J_{\nu}(z)\right|$. Using the series expansion (7.2), and the same argument used for (7.4), we obtain

$$
\left|J_{\nu}(z)\right| \geqslant \frac{\left|\frac{z}{2}\right|^{\nu}}{|\Gamma(\nu+1)|}\left(2-e^{\frac{1}{4}|z|^{2}} \mid \frac{\nu^{2}+1 \mid}{\mid}\right), \quad \nu \neq-1,-2,-3, \ldots
$$

The above bounds imply that, given any $\varepsilon>0$, there exists $z_{0}$ such that, for any $\nu$, if $|z|<\left|z_{0}\right|$ then

$$
\begin{aligned}
\left|J_{\nu}(z)\right| & \leqslant \frac{\left|\frac{z}{2}\right|^{\nu}}{|\Gamma(\nu+1)|}(1+\varepsilon) ; \\
\text { and }\left|J_{\nu}(z)\right| & \geqslant \frac{\left|\frac{z}{2}\right|^{\nu}}{|\Gamma(\nu+1)|}(1-\varepsilon) .
\end{aligned}
$$


If $\nu$ is a negative integer, upper and lower bounds may be deduced using

$$
J_{\nu}(z)=(-1)^{\nu} J_{|\nu|}(z) .
$$

Upper and lower bounds for expressions involving the derivatives of $J_{\nu}(z)$ may be deduced from the recurrence relation:

$$
J_{\nu}^{\prime}(z)=-J_{\nu+1}(z)+\frac{\nu}{z} J_{\nu}(z)
$$

Now we turn to bounds for $\left|H_{\nu}^{(1)}(z)\right|$. These are obtained using the following relation

$$
H_{\nu}^{(1)}(z)=i \csc (\nu \pi)\left\{e^{-\nu \pi i} J_{\nu}(z)-J_{-\nu}(z)\right\} .
$$

Note that we are only interested in the case $\nu \geqslant 0$. Thus, when $\nu>0$ and $\nu \notin \mathbb{Z}$ :

$$
\begin{aligned}
& \left|H_{\nu}^{(1)}(z)\right| \leqslant \frac{\Gamma(\nu)}{\pi}\left|\frac{z}{2}\right|^{-\nu} e^{\frac{\frac{1}{4}|z|^{2}}{\left|\nu_{1}+1\right|}}\left(1+\frac{|\Gamma(1-\nu)|}{\Gamma(1+\nu)}\left|\frac{z}{2}\right|^{2 \nu}\right), \\
& \left|H_{\nu}^{(1)}(z)\right| \geqslant \frac{\Gamma(\nu)}{\pi}\left|\frac{z}{2}\right|^{-\nu}\left(2-e^{\frac{\frac{1}{4}|z|^{2}}{\left|\nu_{1}+1\right|}}\right)\left(1-\frac{|\Gamma(1-\nu)|}{\Gamma(1+\nu)}\left|\frac{z}{2}\right|^{2 \nu}\right) .
\end{aligned}
$$

Here $\left|\nu_{1}+1\right|=\min \{|\nu+1|,|\nu+2|,|\nu+3|, \ldots,|-\nu+1|,|-\nu+2|,|-\nu+3|, \ldots\}$.

For $\nu \in \mathbb{Z}^{+}$, we use the relation:

$$
H_{n}^{(1)}(z)=J_{n}(z)+i Y_{n}(z)
$$

where $Y_{n}(z)$ is the Bessel function of the second kind with series expansion:

$$
\begin{aligned}
Y_{n}(z)= & -\frac{\left(\frac{z}{2}\right)^{-n}}{\pi} \sum_{k=0}^{n-1} \frac{(n-k-1) !}{k !}\left(\frac{z^{2}}{4}\right)^{k}+\frac{2}{\pi} \ln \left(\frac{z}{2}\right) J_{n}(z) \\
& -\frac{\left(\frac{z}{2}\right)^{n}}{\pi} \sum_{k=0}^{\infty} \frac{\psi(k+1)+\psi(n+k+1)}{k !(n+k) !}\left(-\frac{z^{2}}{4}\right)^{k}, n \geqslant 1
\end{aligned}
$$

with the Digamma function $\psi(n)=\frac{\Gamma^{\prime}(n)}{\Gamma(n)}$ given by:

$$
\psi(1)=-\gamma, \quad \psi(n)=-\gamma+\sum_{k=1}^{n-1} \frac{1}{k} \quad n \geqslant 2,
$$

where $\gamma$ is Euler's constant.

Noting that $(n-k-1) ! \leqslant \Gamma(n)$ and $\frac{\psi(k+1)+\psi(n+k+1)}{(n+k) !} \leqslant 2 \Gamma(n)$ for $n \geqslant 1$, we get the following bounds:

$$
\begin{aligned}
\left|Y_{n}(z)\right| \leqslant & \frac{\Gamma(n)}{\pi}\left|\frac{z}{2}\right|^{-n} e^{\frac{1}{4}|z|^{2}}\left\{1+\frac{2}{\Gamma(n)}\left|\frac{z}{2}\right|^{2 n}(1+\right. \\
& \left.\left.+\frac{1}{\Gamma(n+1)}\left|\ln \frac{z}{2}\right| e^{|\Im(z)|-\frac{1}{4}|z|^{2}}\right)\right\} \quad n \geqslant 1 \\
\left|Y_{n}(z)\right| \geqslant & \frac{\Gamma(n)}{\pi}\left|\frac{z}{2}\right|^{-n}\left\{\left(2-e^{\frac{1}{4}|z|^{2}}\right)-\frac{2}{\Gamma(n)}\left|\frac{z}{2}\right|^{2 n} e^{\frac{1}{4}|z|^{2}}-\right. \\
& \left.-\frac{2}{\Gamma(n) \Gamma(n+1)}\left|\ln \frac{z}{2}\right|\left|\frac{z}{2}\right|^{2 n} e^{|\Im(z)|}\right\} \quad n \geqslant 1 .
\end{aligned}
$$


The corresponding inequalities for $H_{n}^{(1)}(z)$ are:

$$
\begin{aligned}
\left|H_{n}^{(1)}(z)\right| \leqslant & \frac{\Gamma(n)}{\pi}\left|\frac{z}{2}\right|^{-n} e^{\frac{1}{4}|z|^{2}}\left\{1+\frac{2}{\Gamma(n)}\left|\frac{z}{2}\right|^{2 n}(1+\right. \\
& \left.\left.+\left(\left|\ln \frac{z}{2}\right|+\frac{\pi}{2}\right) \frac{1}{\Gamma(n+1)} e^{|\Im(z)|-\frac{1}{4}|z|^{2}}\right)\right\}, \quad n \geqslant 1 \\
\left|H_{n}^{(1)}(z)\right| \geqslant & \frac{\Gamma(n)}{\pi}\left|\frac{z}{2}\right|^{-n}\left\{\left(2-e^{\frac{1}{4}|z|^{2}}\right)-\frac{2}{\Gamma(n)}\left|\frac{z}{2}\right|^{2 n} e^{\frac{1}{4}|z|^{2}}-\right. \\
& \left.-\left(2\left|\ln \frac{z}{2}\right|+\pi\right) \frac{1}{\Gamma(n) \Gamma(n+1)}\left|\frac{z}{2}\right|^{2 n} e^{|\Im(z)|}\right\} \quad n \geqslant 1 .
\end{aligned}
$$

The above bounds imply that, given any $\varepsilon>0$, there exists $z_{0}$ such that, for any $\nu>0$, if $|z|<\left|z_{0}\right|$ then

$$
\begin{aligned}
\left|H_{\nu}^{(1)}(z)\right| & \leqslant \frac{\Gamma(\nu)}{\pi}\left|\frac{z}{2}\right|^{-\nu}(1+\varepsilon) \\
\text { and }\left|H_{\nu}^{(1)}(z)\right| & \geqslant \frac{\Gamma(\nu)}{\pi}\left|\frac{z}{2}\right|^{-\nu}(1-\varepsilon) .
\end{aligned}
$$

For $n=0$, we use the following series expansion for $Y_{0}(z)$ :

$$
\begin{aligned}
Y_{0}(z)= & \frac{2}{\pi}\left\{\ln \left(\frac{z}{2}\right)+\gamma\right\} J_{0}(z) \\
& +\frac{2}{\pi}\left\{\frac{z^{2}}{4}-\left(1+\frac{1}{2}\right) \frac{\left(\frac{1}{4} z^{2}\right)^{2}}{(2 !)^{2}}-\left(1+\frac{1}{2}+\frac{1}{3}\right) \frac{\left(\frac{1}{4} z^{2}\right)^{3}}{(3 !)^{2}}-\cdots\right\} .
\end{aligned}
$$

Noting that $1+\frac{1}{2}+\cdots+\frac{1}{n} \leqslant n$ !, we find that the term in the second bracket of $(7.22)$ is bounded in absolute value by $e^{\frac{1}{4}|z|^{2}}-1$. Hence we get the following bounds for $Y_{0}(z)$ :

$$
\begin{aligned}
& \left|Y_{0}(z)\right| \leqslant \frac{2}{\pi}|\ln z|\left\{\left|J_{0}(z)\right|+\frac{\left|J_{0}(z)\right|}{|\ln z|}(\ln 2+\gamma)+\frac{e^{\frac{1}{4}|z|^{2}}-1}{|\ln z|}\right\} ; \\
& \left|Y_{0}(z)\right| \geqslant \frac{2}{\pi}|\ln z|\left\{\left|J_{0}(z)\right|-\frac{\left|J_{0}(z)\right|}{|\ln z|}(\ln 2+\gamma)-\frac{e^{\frac{1}{4}|z|^{2}}-1}{|\ln z|}\right\} .
\end{aligned}
$$

Then, using (7.4) and (7.5) for the case $\nu=0$, we obtain the corresponding bounds for $H_{0}^{(1)}(z)$ :

$$
\begin{aligned}
& \left|H_{0}^{(1)}(z)\right| \leqslant \frac{2}{\pi}|\ln z| e^{\frac{1}{4}|z|^{2}}\left\{1+\frac{1}{|\ln z|}\left(\ln 2+\gamma+\frac{\pi}{2}\right)+\frac{1-e^{-\frac{1}{4}|z|^{2}}}{|\ln z|}\right\} \\
& \left|H_{0}^{(1)}(z)\right| \geqslant \frac{2}{\pi}|\ln z|\left\{\left(2-e^{\frac{1}{4}|z|^{2}}\right)-\frac{e^{\frac{1}{4}|z|^{2}}}{|\ln z|}\left(\ln 2+\gamma+\frac{\pi}{2}\right)-\frac{e^{\frac{1}{4}|z|^{2}}-1}{|\ln z|}\right\} .
\end{aligned}
$$

Now, for fixed $\arg (z)$, we know that $|\ln z| \geqslant \ln |z|$, and that for any $\varepsilon^{\prime}>0,|\ln z| \leqslant$ $\ln |z|\left(1+\varepsilon^{\prime}\right)$ if $|z|$ is small enough. This implies that for any $\varepsilon>0$, there exists $z_{0}$ such that, if $|z|<\left|z_{0}\right|$, then:

$$
\begin{aligned}
& \left|H_{0}^{(1)}(z)\right| \leqslant \frac{2}{\pi} \ln |z|(1+\varepsilon) \\
& \left|H_{0}^{(1)}(z)\right| \geqslant \frac{2}{\pi} \ln |z|(1-\varepsilon) .
\end{aligned}
$$


Next we obtain bounds for the Wronskians that appear in the expressions for the constants $A_{m, R}^{(1)}(k), A_{m, R}^{(2)}(k), B_{m, R}^{(1)}(k)$, and $B_{m, R}^{(2)}(k)$ which are defined in Note 2 of Appendix 1.

Using (7.9) and (7.10), we can write

$$
\begin{aligned}
W\left[H_{\nu}^{(1)}(z), J_{\nu^{\prime}}(z)\right]= & H_{\nu}^{(1)}(z) J_{\nu^{\prime}}^{\prime}(z)-H_{\nu}^{(1) \prime}(z) J_{\nu^{\prime}}(z) \\
= & \frac{i}{\sin (\nu \pi)}\left(\left\{e^{-\nu \pi i} J_{\nu}(z)-J_{-\nu}(z)\right\} J_{\nu^{\prime}}^{\prime}(z)\right. \\
& \left.-\left\{e^{-\nu \pi i} J_{\nu}^{\prime}(z)-J_{-\nu}^{\prime}(z)\right\} J_{\nu}(z)\right) \\
= & \frac{i}{\sin (\nu \pi)}\left(-\frac{\nu^{\prime}+\nu}{z} J_{\nu^{\prime}}(z) J_{-\nu}(z)+J_{\nu^{\prime}+1}(z) J_{-\nu}(z)\right. \\
& -J_{\nu^{\prime}}(z) J_{-\nu+1}(z)+e^{-i \pi \nu}\left\{\frac{\nu^{\prime}-\nu}{z} J_{\nu^{\prime}}(z) J_{\nu}(z)\right. \\
& \left.\left.-J_{\nu^{\prime}+1}(z) J_{\nu}(z)+J_{\nu^{\prime}}(z) J_{\nu+1}(z)\right\}\right) .
\end{aligned}
$$

From the bounds derived above, we obtain

$$
\begin{aligned}
\left|W\left[H_{\nu}^{(1)}(z), J_{\nu^{\prime}}(z)\right]\right| \geqslant & \frac{\left(\nu^{\prime}+\nu\right) \Gamma(\nu)}{2 \pi \Gamma\left(\nu^{\prime}+1\right)}\left|\frac{z}{2}\right|^{\nu^{\prime}-\nu-1}\left\{\left(2-e^{\frac{\frac{1}{4}|z|^{2}}{\nu^{\prime}+1}}\right)\left(2-e^{\frac{\frac{1}{4}|z|^{2}}{\left|\nu_{1}+1\right|}}\right)-\right. \\
& -\frac{2 e^{|\Im(z)|}}{\nu^{\prime}+\nu}\left(\frac{e^{\frac{\frac{1}{4}|z|^{2}}{\nu_{1}+1}}}{\nu^{\prime}+1}\left|\frac{z}{2}\right|^{2}+\frac{e^{\frac{1}{4}|z|^{2}}}{|1-\nu|}\left|\frac{z}{2}\right|^{2}+\frac{\left|\nu^{\prime}+\nu\right| e^{|\Im(z)|}}{2 \Gamma(\nu+1)}\left|\frac{z}{2}\right|^{2 \nu}\right. \\
& \left.\left.+\frac{|\Gamma(1-\nu)| e^{|\Im(z)|}}{\Gamma(\nu+2)}\left|\frac{z}{2}\right|^{2 \nu+2}+\frac{|\Gamma(1-\nu)| e^{|\Im(z)|}}{\left(\nu^{\prime}+1\right) \Gamma(\nu+1)}\left|\frac{z}{2}\right|^{2 \nu+2}\right)\right\}
\end{aligned}
$$

for $\nu, \nu^{\prime}>0, \nu \notin \mathbb{Z}$.

Since $\Gamma(1-\nu)=\frac{\pi}{\sin (\pi \nu) \Gamma(\nu)}$, then for any $\varepsilon_{0}>0$ there exist $z_{0}$ and $\tilde{\nu}_{0}$ such that for $|z|<\left|z_{0}\right|$ and $\nu^{\prime}, \nu>\tilde{\nu}_{0}$

$$
\left|W\left[H_{\nu}^{(1)}(z), J_{\nu^{\prime}}(z)\right]\right| \geqslant \frac{\left(\nu^{\prime}+\nu\right) \Gamma(\nu)}{2 \pi \Gamma\left(\nu^{\prime}+1\right)}\left|\frac{z}{2}\right|^{\nu^{\prime}-\nu-1}\left(1-\varepsilon_{0}\right) .
$$

Using a similar argument to that used in (7.30), as well as the relation

$$
H_{\nu}^{(1) \prime}(z)=-H_{\nu+1}^{(1)}(z)+\frac{\nu}{z} H_{\nu}^{(1)}(z)
$$

we can write

$$
\begin{aligned}
W\left[H_{\nu}^{(1)}(z), H_{n}^{(1)}(z)\right]= & H_{\nu}^{(1)}(z) H_{n}^{(1) \prime}(z)-H_{\nu}^{(1) \prime}(z) H_{n}^{(1)}(z) \\
= & \frac{i}{\sin (\nu \pi)}\left(J_{-\nu}(z) H_{n+1}^{(1)}(z)-\frac{n+\nu}{z} J_{-\nu}(z) H_{n}^{(1)}(z)\right. \\
& -J_{1-\nu}(z) H_{n}^{(1)}(z)+e^{-i \pi \nu}\left\{\frac{n-\nu}{z} J_{\nu}(z) H_{n}^{(1)}(z)\right. \\
& \left.\left.-J_{\nu}(z) H_{n+1}^{(1)}(z)+J_{\nu+1}(z) H_{n}^{(1)}(z)\right\}\right) .
\end{aligned}
$$


Let $n \in \mathbb{Z}^{+}, \nu>0$, and $\nu \notin \mathbb{Z}$. Using the relation $H_{n+1}^{(1)}(z)=\frac{2 n}{z} H_{n}^{(1)}(z)-H_{n-1}^{(1)}(z)$, and the bounds in (7.4) and (7.11), one can see that for any $\varepsilon_{1}>0$ there exist $z_{1}$ and $n_{1}$ such that for $|z|<\left|z_{1}\right|$ and $\nu, n>n_{1}$,

$$
\begin{aligned}
\left|J_{-\nu}(z)\left(H_{n+1}^{(1)}(z)-\frac{n+\nu}{z} H_{n}^{(1)}(z)\right)\right| & \leqslant \frac{\nu-n}{2 \pi} \frac{\Gamma(n)}{|\Gamma(1-\nu)|}\left|\frac{z}{2}\right|^{-n-\nu-1}\left(1+\varepsilon_{1}\right) ; \\
\left|J_{1-\nu}(z) H_{n}^{(1)}(z)\right| & \leqslant \frac{\Gamma(n)}{\pi|\Gamma(2-\nu)|}\left|\frac{z}{2}\right|^{-n-\nu+1}\left(1+\varepsilon_{1}\right) ; \\
\left|\frac{n-\nu}{z} J_{\nu}(z) H_{n}^{(1)}(z)\right| & \leqslant \frac{|n-\nu| \Gamma(n)}{2 \pi \Gamma(\nu+1)}\left|\frac{z}{2}\right|^{-n+\nu-1}\left(1+\varepsilon_{1}\right) ; \\
\left|J_{\nu}(z) H_{n+1}^{(1)}(z)\right| & \leqslant \frac{\Gamma(n+1)}{\pi \Gamma(\nu+1)}\left|\frac{z}{2}\right|^{-n+\nu-1}\left(1+\varepsilon_{1}\right) ; \\
\left|J_{\nu+1}(z) H_{n}^{(1)}(z)\right| & \leqslant \frac{\Gamma(n)}{\pi \Gamma(\nu+2)}\left|\frac{z}{2}\right|^{-n+\nu+1}\left(1+\varepsilon_{1}\right)
\end{aligned}
$$

The above results imply that for $|z|<\left|z_{1}\right|$ and $\nu, n>n_{1}$,

$$
\begin{aligned}
& \left|W\left[H_{\nu}^{(1)}(z), H_{n}^{(1)}(z)\right]\right| \leqslant \frac{|\nu-n|}{2 \pi^{2}} \Gamma(n) \Gamma(\nu)\left|\frac{z}{2}\right|^{-n-\nu-1}\left(1+\frac{2}{|\nu-n||1-\nu|}\left|\frac{z}{2}\right|^{2}\right. \\
& +\frac{|\Gamma(1-\nu)|}{\Gamma(\nu+1)}\left|\frac{z}{2}\right|^{2 \nu}+\frac{2 n \mid \Gamma(1-\nu)}{|\nu-n| \Gamma(\nu+1)}\left|\frac{z}{2}\right|^{2 \nu} \\
& \left.+\frac{2|\Gamma(1-\nu)|}{|\nu-n| \Gamma(\nu+2)}\left|\frac{z}{2}\right|^{2 \nu+2}\right)\left(1+\varepsilon_{1}\right)
\end{aligned}
$$

From this we deduce that for any $\varepsilon_{2}>0$ there exist $z_{2}$ and $n_{2}$ such that for $|z|<\left|z_{2}\right|$ and $\nu, n>n_{2}$

$$
\left|W\left[H_{\nu}^{(1)}(z), H_{n}^{(1)}(z)\right]\right| \leqslant \frac{|\nu-n|}{2 \pi^{2}} \Gamma(n) \Gamma(\nu)\left|\frac{z}{2}\right|^{-n-\nu-1}\left(1+\varepsilon_{2}\right)
$$

Using (7.9), we obtain

$$
\begin{aligned}
W\left[J_{n}(z), J_{\nu}(z)\right] & =J_{n}(z) J_{\nu}^{\prime}(z)-J_{n}^{\prime}(z) J_{\nu}(z) \\
& =\frac{\nu-n}{z} J_{n}(z) J_{\nu}(z)-J_{n}(z) J_{\nu+1}(z)+J_{n+1}(z) J_{\nu}(z) .
\end{aligned}
$$

Then, for $n \in \mathbb{Z}^{+}$, and $\nu \geqslant-\frac{1}{2}$,

$$
\left|W\left[J_{n}(z), J_{\nu}(z)\right]\right| \leqslant \frac{|\nu-n| e^{|\Im(z)|}}{2 \Gamma(n+1) \Gamma(\nu+1)}\left|\frac{z}{2}\right|^{n+\nu-1}\left\{1+\left(\frac{2 n}{\nu+1}+\frac{2}{n+1}\right)\left|\frac{z}{2}\right|^{2}\right\} .
$$

In the case $n=|m|, \nu=|m-\alpha|$, for any $\varepsilon_{4}>0$ there exist $z_{4}$ and $n_{4}$ such that for $|z|<\left|z_{4}\right|$ and $\nu, n>n_{4}$,

$$
\left|W\left[J_{n}(z), J_{\nu}(z)\right]\right| \leqslant \frac{|\nu-n|}{2 \Gamma(n+1) \Gamma(\nu+1)}\left|\frac{z}{2}\right|^{n+\nu-1}\left\{1+\varepsilon_{4}\right\}
$$




\section{References}

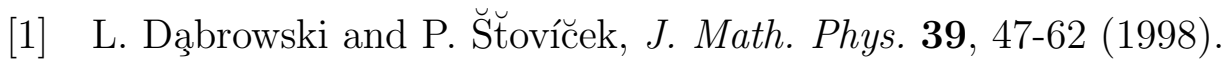

[2] R. Adami and A. Teta, Lett. Math. Phys. 43, 43-53 (1998).

[3] M. Bordag and S. Voropaev, J. Phys. A 26, 7637-7649 (1993).

[4] A. Moroz, Phys. Rev. A 53, 669-694 (1996).

[5] H. Tamura, Rev. Math. Phys. 13, 465-511 (2001).

[6] C.R. Hagen, Phys. Rev. Lett. 64, 503-506 (1990).

[7] S. Albeverio, F. Gesztesy, R. Hoegh-Krohn and H. Holden: Solvable Models in Quantum Mechanics, Springer-Verlag, Heidelberg (1988).

[8] M. Abramowitz and I.A. Stegun: Handbook of Mathematical Functions, Dover Publications, New York (1965).

[9] I.S. Gradshteyn and I.M. Ryzhik: Table of Integrals, Series and Products, Academic Press, New York (1965).

[10] G.N. Watson: A Treatise on the Theory of Bessel Functions, Cambridge University Press, London (1944). 